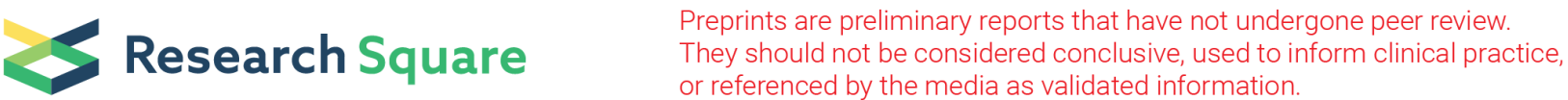 \\ Influences of the Workpiece Pose Errors on Gear Skiving Accuracy
}

\section{Xiaochuan Lin}

Nanjing Tech University

rongjing hong ( $\nabla$ rjhnjut@163.com )

Nanjing Tech University https://orcid.org/0000-0002-6402-9158

\section{Yawen Wang}

University of Texas at Arlington

\section{Yun Peng}

Nanjing University of Aeronautics and Astronautics

\section{Xiaolei Ren}

University of Texas at Arlington

\section{Research Article}

Keywords: Gear skiving accuracy, workpiece pose errors, numerical analysis, error measurement, PSO algorithm

Posted Date: August 13th, 2021

DOl: https://doi.org/10.21203/rs.3.rs-793798/v1

License: (c) (i) This work is licensed under a Creative Commons Attribution 4.0 International License. Read Full License

Version of Record: A version of this preprint was published at The International Journal of Advanced Manufacturing Technology on February 1st, 2022. See the published version at https://doi.org/10.1007/s00170-022-08813-0. 


\section{Influences of the workpiece pose errors on gear skiving accuracy \\ Xiaochuan Lin ${ }^{\mathrm{a}, \mathrm{b}}$, Rongjing Hong*a,b , Yawen Wang ${ }^{\mathrm{c}}$, Yun Peng ${ }^{\mathrm{d}}$, Xiaolei Ren}

Author information:

First Author: Xiaochuan Lin

a School of Mechanical and Power Engineering, Nanjing Tech University, Nanjing, 211816, China

b Jiangsu Key Laboratory of Digital Manufacturing for Industrial Equipment and Control

Technology, Nanjing Tech University, Nanjing, 211816, China

E-mail: xiaochuan_lin@163.com

Fax: +86 025-53533233-820

TEL: +8615062286013

Address: No.30, Puzhu Sothern Road, Pukou District, Nanjing, Jiangsu, China

*Corresponding Author: Rongjing Hong

a School of Mechanical and Power Engineering, Nanjing Tech University, Nanjing, 211816, China

b Jiangsu Key Laboratory of Digital Manufacturing for Industrial Equipment and Control

Technology, Nanjing Tech University, Nanjing, 211816, China

E-mail:rjhnjut@163.com

Fax: +86 025-53533233-820

TEL: +8613905177495

Address: No.30, Puzhu Sothern Road, Pukou District, Nanjing, Jiangsu, China

Co-author: Yawen Wang

c Department of Mechanical and Aerospace Engineering, University of Texas at Arlington, Texas

76019, USA

E-mail: yawen.wang@uta.edu

Fax: +15139178577

TEL: +15139178577

Address: $701 \mathrm{~S}$ Nedderman Dr, Arlington, Texas, USA

Co-author: Yun Peng

d College of Mechanical and Electrical Engineering, Nanjing University of Aeronautics and

Astronautics, Nanjing, 210016, China

E-mail: 1091190621@qq.com

Fax: +86 025-53533233-820

TEL: +8619852831076

Address: No.29, Yudao Road, Qinhuai District, Nanjing, Jiangsu, China

Co-author: Xiaolei Ren

e Department of Computer Science and Engineering, University of Texas at Arlington, Texas 76019, USA

E-mail: xiaolei.ren@uta.edu

Fax: +18172722011

TEL: +18172722011

Address: $701 \mathrm{~S}$ Nedderman Dr, Arlington, Texas, USA

Acknowledgments: This work was supported by the National Natural Science Foundation of China (Grant No. 51635003) and the Postgraduate Research \& Practice Innovation Program of Jiangsu Province (Grant No.KYCX18_1092). Besides, the authors would like to express their sincere appreciation to them for providing research funding. 


\title{
Influences of the workpiece pose errors on gear skiving accuracy
}

\begin{abstract}
This paper investigates the influence of the workpiece pose errors on gear skiving accuracy. First, based on the theory of homogeneous coordinate transformation, the mapping relationship between the tooth flank of the workpiece and the skiving tool is established according to the principle of gear skiving. Second, the workpiece pose errors are defined, and the relationship between each workpiece pose error and cutting depth is analyzed. According to the kinematic chain of the machine tool, a method for measuring the workpiece pose errors based on actual inverse kinematics is proposed. Third, the tooth deviations from individual workpiece pose errors, and the coupling workpiece pose errors are studied. The sensitive errors are obtained from the tooth deviation by numerical simulation. Finally, the numerical analysis is verified by gear skiving experiments, and the proposed method can be used to identify the possible workpiece pose errors. Furthermore, the PSO algorithm is introduced to guide the workpiece setting pose errors in the gear skiving process.
\end{abstract}

Keywords: Gear skiving accuracy, workpiece pose errors, numerical analysis, error measurement, PSO algorithm.

\section{Introduction}

Gear skiving is a modern and productive method for machining internal and external cylindrical gears. In recent years, gear skiving has attracted the attention of researchers and manufacturers. It has better efficiency and accuracy than the traditional gear hobbing and gear shaping of small module gears and can machine the internal gear without a relief groove. Moreover, gear skiving technology aims to be used in the gear finishing process to reduce the gear distortion errors caused by heat treatment. Therefore, the potential advantages of gear skiving drive some well-known machine tool manufacturers to produce high-end gear skiving machines [1-4].

Many scholars have made progress on the design and manufacture technologies of the skiving tool and cutting mechanism. A variety of new high-performance skiving tool has been developed, such as super cylindrical skiving tool [5-7], assembled skiving tool [8-10], and super-skiving tool designed by MHI [1]. Li and Chen et al. [11,12] researched the basic principle of the skiving technology and proposed a design method of error-free spur skiving tool for internal helical gear. Then, Guo and Hong et al. [13-15] established the mathematical models for designing spur and helical skiving tool and researched the cutting mechanism of the gear skiving to optimize the tool design. In addition, skiving strategies have also been optimized to improve the skiving tool life, machining accuracy, and efficiency. Marx and Vogel [16] proposed a semi-completing skiving machining strategy. Kreschel and Kobialka [17] proposed a multiple-feed skiving technology. Guo et al. [18] combined the characteristics of skiving strategies in $[16,17]$ and proposed a multiple-step feed machining technology to effectively reduce the cutting force, improve the tool life and the cutting performance. Uriu et al. [19] analyzed the relationship between the shaft angle and the cutting speed to determine the tool setting parameters, which can effectively improve the accuracy of the tooth profile of the tool. Tsai et al. [20] analyzed the main factors determining the linear cutting speed of the power-skiving tool and a modified D-H notation is used to evaluate the error characteristics of the machine tool system, and the power-skiving is realized in the six-axis CNC turn-mill machine center.

Although skiving technologies have developed rapidly in recent years, the research on the influence of geometric errors on the accuracy of gear skiving is sparse. Most of the existing researches focused on tool eccentricity. Guo et al. [21] analyzed the influences of tool setting on gear skiving accuracy on profile accuracy, the spiral deviation, and tooth surface quality. Ren et al. [22] studied the influence of different cutter eccentricities on tooth surface roughness under different feed rates by numerical analysis. Yang et al. [23] established the tooth flank equation with errors and the mapping 
relationship between the geometric pose errors and profile normal errors. The sensitivity of profile normal errors to pose errors is analyzed. However, there is no detailed study on the influence of workpiece pose errors caused by setting, cutting force, and heat, on the gear skiving accuracy. In general, gear skiving is usually used for machining gear with a small module (less than $5 \mathrm{~mm}$ ). The smaller the gear module, the more significant influence of the workpiece pose errors on the tooth deviations. Therefore, it is necessary to study the relationship between workpiece pose errors and gear skiving accuracy.

In this paper, the influences of the workpiece pose errors on gear skiving accuracy are studied, including profile deviations, helix deviations, and pitch deviations. Firstly, the mapping relationship between the tooth flank of the workpiece and the skiving tool is established. Secondly, the pose errors of the workpiece are identified, and the regulations of the cutting depth under different pose errors in the gear skiving process are analyzed. Then, a method for measuring the workpiece pose errors based on actual inverse kinematics is proposed. Thirdly, the influences of individual workpiece pose error and the coupling workpiece pose errors on accuracy are studied. Finally, a series of experiments are performed to verify the numerical analysis. The PSO algorithm is used to predict the maximum workpiece setting pose errors in the gear skiving process.

\section{Principle of gear skiving}

During the gear skiving processing, the tool and workpiece are placed so that their rotating axes form a crossed axes angle. They rotate synchronously to generate a sliding motion at the contact point, thereby performing cutting [1].

\subsection{Motion model of gear skiving}

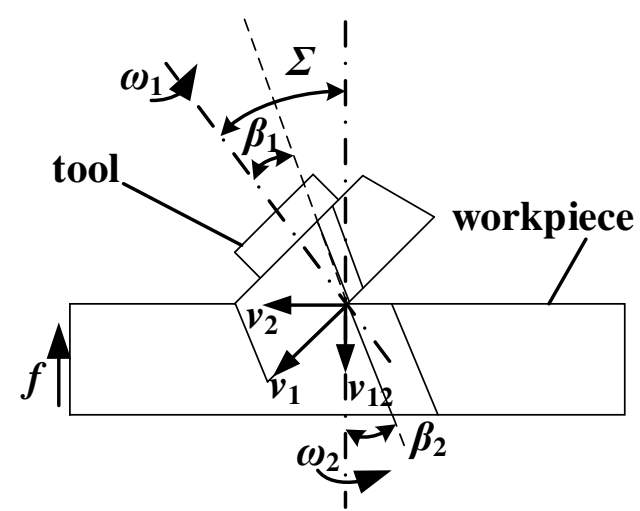

Fig. 1. Kinematical principle of gear skiving.

The kinematical principle of gear skiving is shown in Fig. 1. There is a shaft angle $\Sigma$ between the tool axis and the workpiece axis. The tool and workpiece rotate around their axes with angular velocity $\omega_{1}$ and $\omega_{2}$, respectively. At the same time, the workpiece has an axial feed $f$ along the workpiece axis. Therefore, the angular velocity $\omega_{2}$ can be obtained as follows:

$$
\omega_{2}=\frac{z_{t}}{z_{g}} \omega_{1}+\frac{f}{p}
$$

where the $z_{t}$ and $z_{g}$ are the tooth number of the tool and workpiece, respectively, and $p$ is the helix parameter which can be express as

$$
p=\frac{p_{z}}{2 \pi}
$$

where $p_{z}$ is the helix lead on the reference cylinder of the workpiece, $p_{z}=\frac{\pi d_{g}}{\tan \beta_{g}}$. 


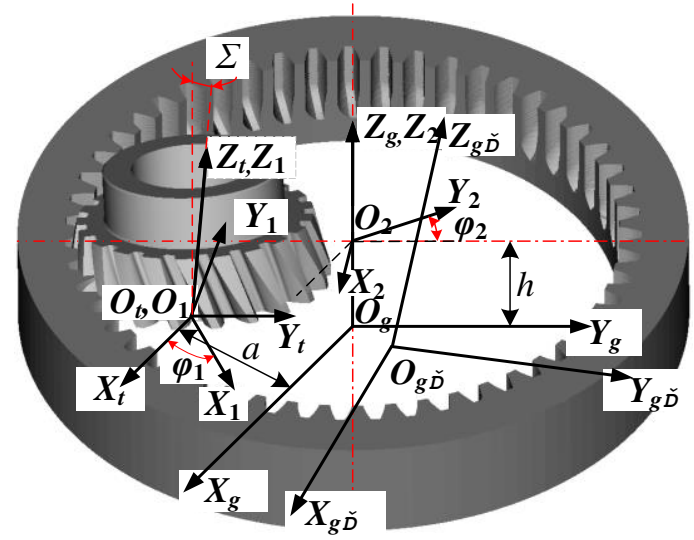

Fig. 2 The definition of the coordinate system.

As shown in Fig. 2, $S_{t}\left(O_{t}-X_{t} Y_{t} Z_{t}\right)$ is the fixed coordinate system of the tool that rotates around the $Z_{t}$-axis. $S_{g}\left(O_{g}-X_{g} Y_{g} Z_{g}\right)$ is the fixed coordinate system of the workpiece that rotates around the $Z_{g}$-axis. $S_{1}\left(O_{1}-X_{1} Y_{1} Z_{1}\right)$ and $S_{2}\left(O_{2}-X_{2} Y_{2} Z_{2}\right)$ are rigidly attached to the tool and workpiece, respectively. The initial positions of $S_{1}$ and $S_{2}$ have coincided with the $S_{t}$ and $S_{g}$, respectively. $S_{g}{ }^{\prime}\left(O_{g}{ }^{\prime} X_{g}, Y_{g}{ }^{\prime} Z_{g}{ }^{\prime}\right)$ is the coordinate system attached to the tool, which has the workpiece pose errors.

According to the homogeneous coordinate transformation, the transformation matrices from $S_{t}$ to $S_{g}$ can be obtained:

$$
\left[\begin{array}{c}
x_{g} \\
y_{g} \\
z_{g} \\
1
\end{array}\right]=\mathbf{M}_{g_{2}} \mathbf{M}_{21} \mathbf{M}_{1 t}\left[\begin{array}{c}
x_{t} \\
y_{t} \\
z_{t} \\
1
\end{array}\right]
$$

where $\mathbf{M}_{g 2}$ is the transfer-matrix from $S_{2}$ to $S_{g}, \mathbf{M}_{21}$ is the transfer-matrix from $S_{1}$ to $S_{2}, \mathbf{M}_{1 t}$ is the transfer-matrix from $S_{t}$ to $S_{1}$.

$$
\begin{aligned}
\mathbf{M}_{g 2} & =\left[\begin{array}{cccc}
\cos \left(\varphi_{2}\right) & -\sin \left(\varphi_{2}\right) & 0 & 0 \\
\sin \left(\varphi_{2}\right) & \cos \left(\varphi_{2}\right) & 0 & 0 \\
0 & 0 & 1 & -h \\
0 & 0 & 0 & 1
\end{array}\right], \mathbf{M}_{21}=\left[\begin{array}{cccc}
\cos (\Sigma) & 0 & \sin (\Sigma) & 0 \\
0 & 1 & 0 & a \\
-\sin (\Sigma) & 0 & \cos (\Sigma) & 0 \\
0 & 0 & 0 & 1
\end{array}\right], \\
\mathbf{M}_{1 t} & =\left[\begin{array}{cccc}
\cos \left(\varphi_{1}\right) & -\sin \left(\varphi_{1}\right) & 0 & 0 \\
\sin \left(\varphi_{1}\right) & \cos \left(\varphi_{1}\right) & 0 & 0 \\
0 & 0 & 1 & 0 \\
0 & 0 & 0 & 1
\end{array}\right] .
\end{aligned}
$$

where $\varphi_{1}$ and $\varphi_{2}$ are the rotation angles of the tool and workpiece around the $Z_{t}$-axis and $Z_{g}$-axis, respectively, $\varphi_{1}=\omega_{1} t, \varphi_{2}=\omega_{2} t$, and $t$ is the cutting time. The shaft angle $\Sigma=\left|\beta_{1} \pm \beta_{2}\right|, h=f t$ is the width of the workpiece, and $a$ is the center distance. Let $\mathbf{M}_{g t}=\mathbf{M}_{g_{2}} \mathbf{M}_{21} \mathbf{M}_{1 t}$.

\section{Identification and analysis of workpiece pose errors}

The workpiece pose errors are generated during the workpiece setting process, including 3 position deviation errors and 3 angle deviation errors, as shown in Table 1. According to the research by Ding et al. [24], the Motion-first model and Error-first model can be used to analyze the geometric error of the rotary axis. The Error-first model is preferred for the modeling and definition of geometric 
errors of the rotary axis. Therefore, the Error-first model will be used to define and identify the workpiece pose errors in the gear skiving system.

3.1 Identification of workpiece pose errors

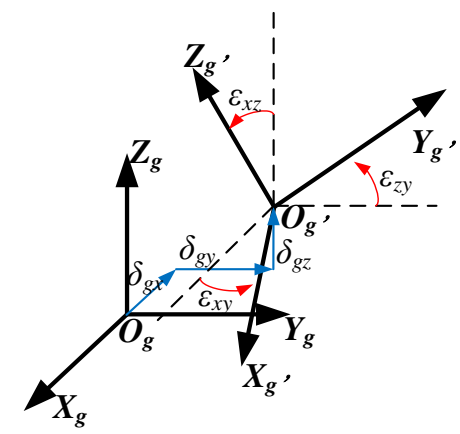

Fig. 3. Pose errors of the workpiece.

As shown in Fig. 3, under the influence of the workpiece pose errors, the coordinate system of the workpiece without errors $S_{g}$ is the theoretical coordinate system, and the coordinate system of the workpiece with error $S_{g}$ is the actual coordinate system. It is assumed that all pose errors are small, and each error is relatively independent in the process of workpiece movement. Table $\mathbf{1}$ shows the definition of workpiece setting pose errors.

Table 1 The workpiece setting pose errors.

\begin{tabular}{llll}
\hline Errors & Geometric description of the error & Errors & Geometric description of the error \\
\hline$\delta_{g x}$ & Position deviation error along $X_{g}$-axis & $\varepsilon_{x z}$ & Angle deviation error around $Y_{g}$-axis \\
$\delta_{g y}$ & Position deviation error along $Y_{g}$-axis & $\varepsilon_{z y}$ & Angle deviation error around $X_{g}$-axis \\
$\delta_{g z}$ & Position deviation error along $Z_{g}$-axis & $\varepsilon_{x y}$ & Angle deviation error around $Z_{g}$-axis \\
\hline
\end{tabular}

For simplicity, abbreviate cosine and sine as " $C$ " and " $S$ ", so the transfer-matrix from $S_{g}$ ' to $S_{g}$ can be expressed as

$$
\begin{aligned}
\mathbf{M}_{g^{\prime} g} & =\left[\begin{array}{cccc}
1 & 0 & 0 & \delta_{g x} \\
0 & 1 & 0 & \delta_{g y} \\
0 & 0 & 1 & \delta_{g z} \\
0 & 0 & 0 & 1
\end{array}\right]\left[\begin{array}{cccc}
C\left(\varepsilon_{x y}\right) & -S\left(\varepsilon_{x y}\right) & 0 & 0 \\
S\left(\varepsilon_{x y}\right) & C\left(\varepsilon_{x y}\right) & 0 & 0 \\
0 & 0 & 1 & 0 \\
0 & 0 & 0 & 1
\end{array}\right]\left[\begin{array}{cccc}
C\left(\varepsilon_{x z}\right) & 0 & S\left(\varepsilon_{x z}\right) & 0 \\
0 & 1 & 0 & 0 \\
-S\left(\varepsilon_{x z}\right) & 0 & C\left(\varepsilon_{x z}\right) & 0 \\
0 & 0 & 0 & 1
\end{array}\right]\left[\begin{array}{cccc}
1 & 0 & 0 & 0 \\
0 & C\left(\varepsilon_{z y}\right) & -S\left(\varepsilon_{z y}\right) & 0 \\
0 & S\left(\varepsilon_{z y}\right) & C\left(\varepsilon_{z y}\right) & 0 \\
0 & 0 & 0 & 1
\end{array}\right] \\
& =\left[\begin{array}{cccc}
C\left(\varepsilon_{x y}\right) C\left(\varepsilon_{x z}\right) & C\left(\varepsilon_{x y}\right) S\left(\varepsilon_{x z}\right) S\left(\varepsilon_{z y}\right)-C\left(\varepsilon_{z y}\right) S\left(\varepsilon_{x y}\right) & S\left(\varepsilon_{x y}\right) S\left(\varepsilon_{z y}\right)+C\left(\varepsilon_{x y}\right) C\left(\varepsilon_{z y}\right) S\left(\varepsilon_{x z}\right) & \delta_{g x} \\
C\left(\varepsilon_{x z}\right) S\left(\varepsilon_{x y}\right) & C\left(\varepsilon_{x y}\right) C\left(\varepsilon_{z y}\right)+S\left(\varepsilon_{x y}\right) S\left(\varepsilon_{x z}\right) S\left(\varepsilon_{z y}\right) & C\left(\varepsilon_{z y}\right) S\left(\varepsilon_{x y}\right) S\left(\varepsilon_{x z}\right)-C\left(\varepsilon_{x y}\right) S\left(\varepsilon_{z y}\right) & \delta_{g y} \\
-S\left(\varepsilon_{x z}\right) & C\left(\varepsilon_{x z}\right) S\left(\varepsilon_{z y}\right) & C\left(\varepsilon_{x z}\right) C\left(\varepsilon_{z y}\right) & \delta_{g z} \\
0 & 0 & 0 & 1
\end{array}\right]
\end{aligned}
$$

Assuming the angle deviation errors $\left\{\varepsilon_{x y}, \varepsilon_{x z}, \varepsilon_{z y}\right\}$ are small, $\cos (\varepsilon) \approx 1$ and $\sin (\varepsilon) \approx \varepsilon$, where $\varepsilon=\varepsilon_{x y}, \varepsilon_{x z}, \varepsilon_{z y}$. By ignoring high-order infinitesimals, the transfer-matrix of Eq.(4) can be represented by

$$
\mathbf{M}_{g^{\prime} g}=\left[\begin{array}{cccc}
1 & -\varepsilon_{x y} & \varepsilon_{x z} & \delta_{g x} \\
\varepsilon_{x y} & 1 & -\varepsilon_{z y} & \delta_{g y} \\
-\varepsilon_{x z} & \varepsilon_{z y} & 1 & \delta_{g z} \\
0 & 0 & 0 & 1
\end{array}\right]
$$

It can be known that the position deviation error $\delta_{g z}$ and the angle deviation error $\varepsilon_{x y}$ only affect the initial position of the workpiece because the direction of the $Z$-axis keeps unchanged during 
the rotation of the workpiece [24]. Therefore, the main workpiece pose errors that affect the skiving accuracy are $\left\{\delta_{g x}, \delta_{g y}, \varepsilon_{x y}, \varepsilon_{x z}\right\}$, and the Eq. (5) can be represented by

$$
\mathbf{M}_{g^{\prime} g}=\left[\begin{array}{cccc}
1 & 0 & \varepsilon_{x z} & \delta_{g x} \\
0 & 1 & -\varepsilon_{z y} & \delta_{g y} \\
-\varepsilon_{x z} & \varepsilon_{z y} & 1 & 0 \\
0 & 0 & 0 & 1
\end{array}\right]
$$

\subsection{Regulations between cutting depth and workpiece pose errors}

According to the principle of gear skiving, skiving can be regarded as the relative movement of the tool and the workpiece. The cutting edge of the tool can be regarded as a curve on the rake face. The tool continuously moves to envelop the tooth flank. Therefore, the target tooth flank is an involute spiral surface, which can be expressed as

$$
r_{g}(u, \theta)=\left[\begin{array}{c}
r_{b} \cos (u+\theta)+r_{b} u \sin (u+\theta) \\
r_{b} \sin (u+\theta)-r_{b} u \cos (u+\theta) \\
p \theta \\
1
\end{array}\right]
$$

where $r_{b}$ is the base circle radius of the workpiece, $u$ is the involute parameter, $\theta$ is the rotation angle of the transverse tooth profile of the target tooth flank around the $Z_{g}$-axis.

The coordinates of the point on the cutting edge of the tool are considered as a function of the independent variable $u$, so the expression of the point on the cutting edge in $S_{t}$ can be obtained as follows:

$$
\boldsymbol{r}_{t}(u)=\left[\begin{array}{llll}
x_{t}(u) & y_{t}(u) & z_{t}(u) & 1
\end{array}\right]^{\mathrm{T}}
$$

When the workpiece pose errors are zero, combining Eqs.(3), (7) and (8), the equation of swept target tooth flank in $S_{g}$ can be obtained as follows:

$$
\boldsymbol{r}_{g}\left(u, \theta, \varphi_{2}\right)=\mathbf{M}_{g t} \boldsymbol{r}_{t}\left(u, \varphi_{1}\right)
$$

When the workpiece pose errors are not zero, substituting Eq. (6) into Eq. (9), the swept target tooth flank in $S_{g}$, can be expressed as:

$$
\boldsymbol{r}_{g^{\prime}}\left(u, \theta, \varphi_{2}, \delta, \varepsilon\right)=\mathbf{M}_{g^{\prime} g} \mathbf{M}_{g t} \boldsymbol{r}_{t}\left(u, \varphi_{1}\right)
$$

where $\delta=\left\{\delta_{g x}, \delta_{g y}\right\}$ and $\varepsilon=\left\{\varepsilon_{x z}, \varepsilon_{z y}\right\}$.

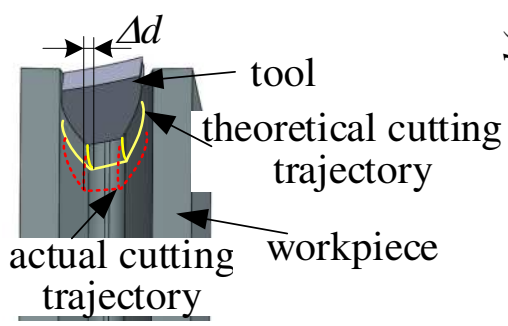

(a)

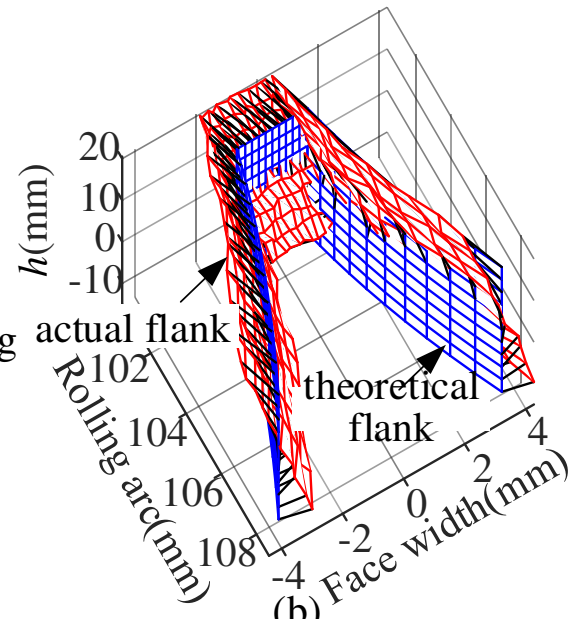

(b)

Fig. 4. Cutting diagram. (a) Cutting trajectory of gear skiving, (b)Normal deviation topography for skived gear. 
Therefore, the trajectory of the skiving process can be obtained. The cutting trajectory of gear skiving in a single tooth flank is shown in Fig. 4 (a). It can be observed that the actual cutting trajectory deviates from the theoretical cutting trajectory due to the workpiece pose errors. The actual flank becomes twisted and reduces the skiving accuracy, as shown in Fig. 4 (b). Furthermore, the cutting depth variation $\Delta d$, the difference between the actual cutting depth and the theoretical one, can be expressed as:

$$
\Delta d=\sqrt{x_{g}{ }^{2}+y_{g}{ }^{2}}-\sqrt{x_{g^{\prime}}{ }^{2}+y_{g^{\prime}}{ }^{2}}
$$

Using the Eq. (11), when all the position deviation errors $\delta$ are set as $0.05 \mathrm{~mm}$ and the angle deviation errors $\varepsilon$ are set as $0.05^{\circ}$, the regulations of the cutting depth $\Delta d$ changing with the workpiece width $h$ and the tooth number of workpiece $z_{g}$ are studied.

As shown in Fig. 5, the influence of the position deviation errors on the cutting depth is a nearfixed value when $z_{g}=1$. Significantly when it is affected by the position deviation error in the $x$ direction, the error value is almost completely mapped on the cutting depth. However, the angle deviation errors present a slope of $\tan \varepsilon$ in affecting the cutting depth, which increases the cutting depth with the increase of workpiece width.

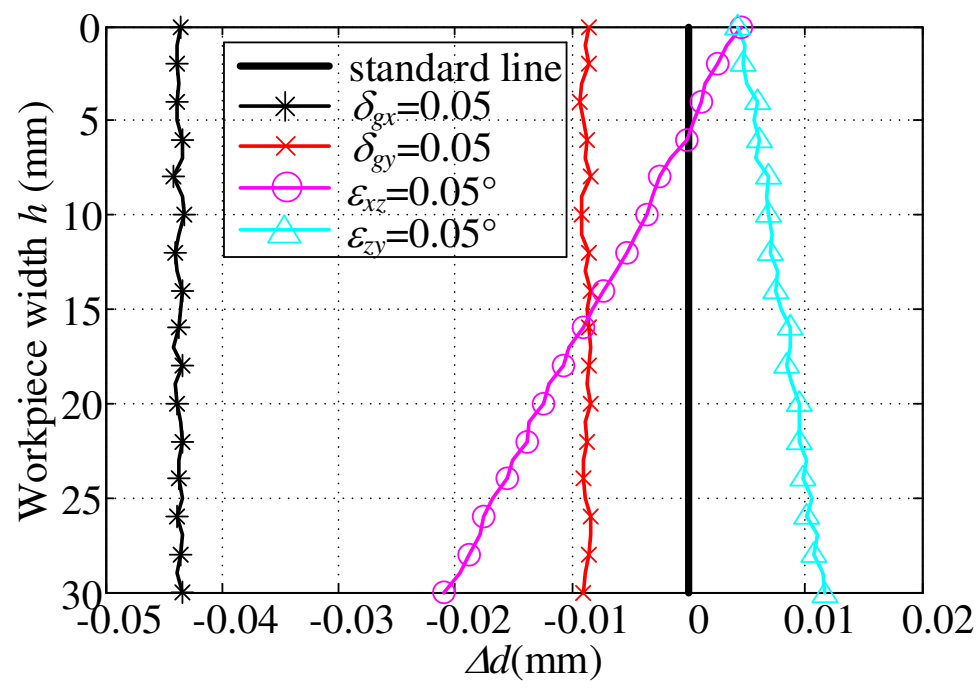

Fig. $5 \Delta d$ change with workpiece width under individual pose error when $z_{g}=1$.

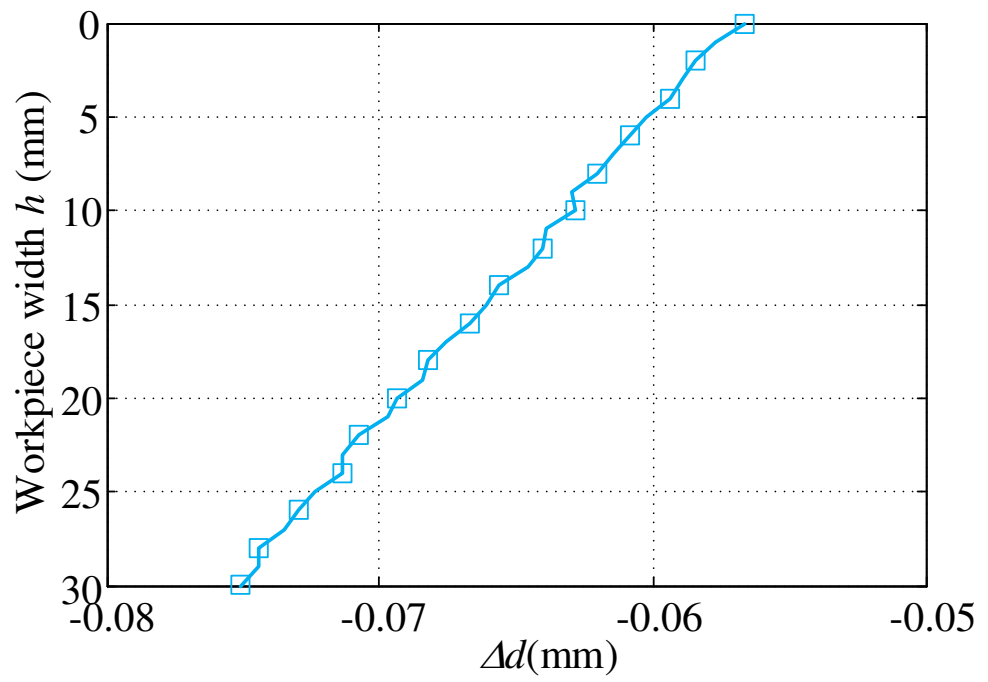

Fig. $6 \Delta d$ change with workpiece width under the coupling pose errors when $z_{g}=1$.

The regulation of the $\Delta d$ changing with workpiece width under the coupling pose errors is shown 
in Fig. 6. Due to the influence of the four pose errors, the cutting depth increases with the increase of workpiece width. The change of the $\Delta d$ under the coupling errors is approximately equal to the linear superposition of the change of each pose error in Fig. 5. In addition, it can be seen from Fig. 5 and Fig. 6 that the curve is not a smooth line but a wavy line. Our analysis is that because the gear skiving is a continuous chip-breaking cutting, the gear flank will have tiny ripples. Moreover, the results of the numerical simulation are visible to match the gear skiving principle well.

The cutting depth $\Delta d$ change with the tooth number of workpiece $z_{g}$ is shown in Fig. 7 . When the workpiece width $h=15 \mathrm{~mm}$, the influences of the position and angle deviation errors on the cutting depth change periodically with the change of tooth number in a cosine or sine curve. The angle deviation errors have a much smaller impact on the $\Delta d$ than the position deviation errors. It shows that $\Delta d$ is mainly affected by the position deviation errors in this position. The influence of the angle deviation errors on $\Delta d$ gradually increases with the change of the workpiece width, as shown in Fig. 5. When the position and angle deviation errors are coupled, the $\Delta d$ is a cosine or sine cycle change curve as the tooth number changes. Its value can be approximately regarded as a linear superposition under the influence of each error. It can be found that the workpiece pose errors are of great significance to improve the skiving accuracy. It is necessary to propose a method for measuring the workpiece pose error during gear skiving. The mapping relationship between the workpiece pose errors and gear skiving accuracy should be studied.

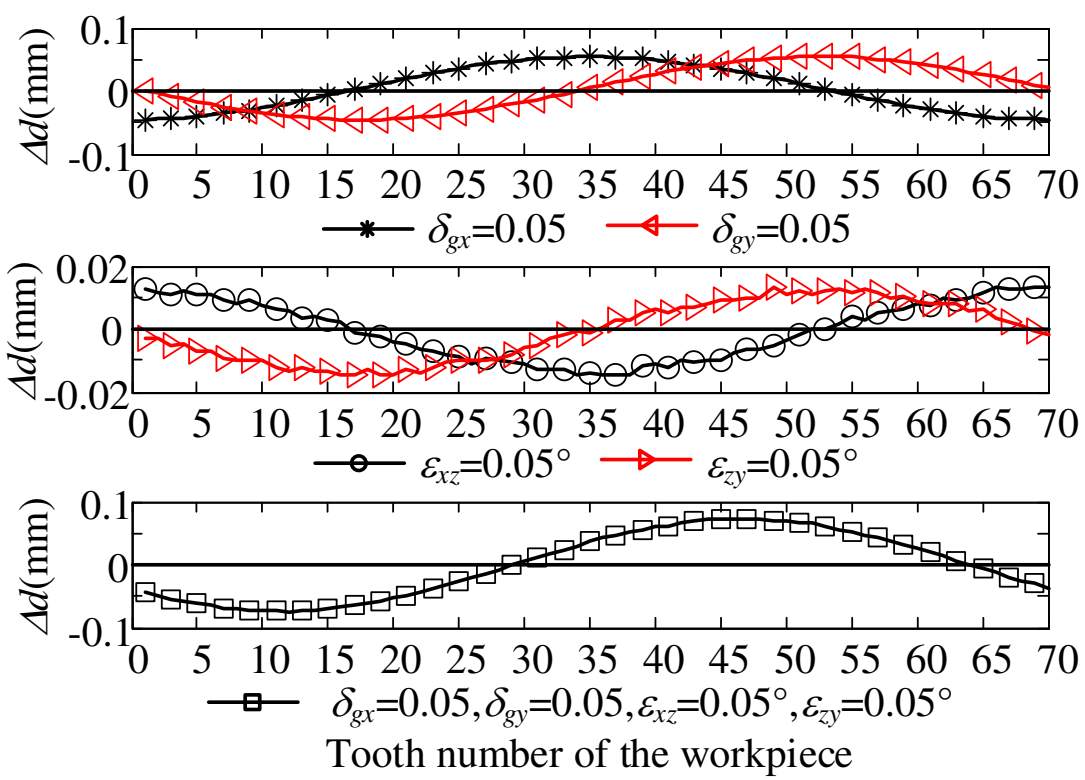

Fig. $7 \Delta d$ change with $z_{g}$ when the workpiece width $h=15$.

\section{Measurement of workpiece pose errors}

To measure the workpiece pose errors quickly, a method for measuring the defined workpiece pose errors $\left\{\delta_{g x}, \delta_{g y}, \varepsilon_{z y}, \varepsilon_{x z}\right\}$ based on actual inverse kinematics is proposed in this paper. The schematic of the gear skiving machine tool used in this paper is shown in Fig. 8 (a). To make the measurement more convenient, the workpiece origin coordinate $O_{g}$ is set as $\left[\begin{array}{llll}0 & 0 & 0 & 1\end{array}\right]^{\mathrm{T}}$, which is also the reference origin of the machine tool. The proposed method uses the tool as the measurement reference in this paper. It is noted that the proposed method employs an on-machine measurement system, which is different from the online measurement system. Therefore, according to Fig. 2 and Fig. 8 , the transformation matrices from $S_{t}$ to $S_{g}$ can be expressed as

$$
\mathbf{M}_{g t}=\left(\mathbf{M}_{x g} \mathbf{M}_{g^{\prime} g}\right)^{-1} \cdot \mathbf{M}_{x t}
$$




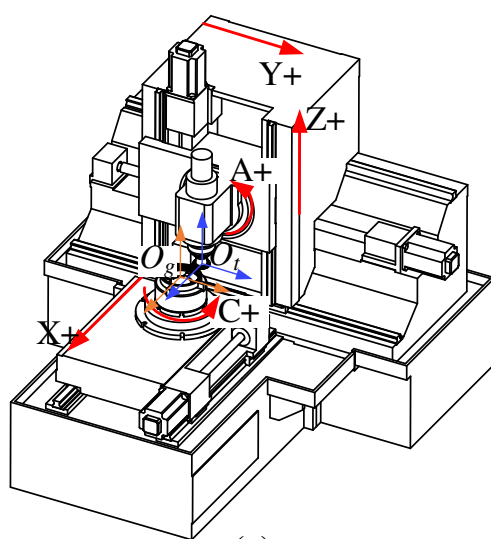

(a)

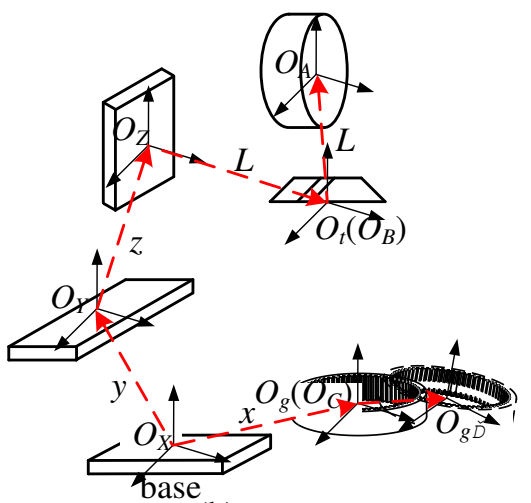

(b)

Fig. 8. Example of gear skiving machine tool, (a) schematic of machine tool, (b) the kinematic chain of machine tool

According to the kinematic chain of the machine tool shown in Fig. 8 (b), substituting Eq. (6) into Eq. (12), the transformation matrices from $S_{t}$ to $S_{g}$ can be obtained as follows:

$$
\begin{aligned}
\mathbf{M}_{g t} & =\left(\mathbf{T}_{X}(x) \mathbf{T}_{Z}(h) \mathbf{R}_{Z}(c)\left(\mathbf{M}_{g^{\prime} g}\right)^{-1}\right)^{-1} \\
& \cdot \mathbf{T}_{Y}(y) \mathbf{T}_{Z}(z) \mathbf{T}_{Z}(L) \mathbf{R}_{X}(a) \mathbf{R}_{Y}(b) \mathbf{T}_{Z}(-L) \\
& =\left[\begin{array}{cccc}
a 11 & a 12 & a 13 & a 14 \\
a 21 & a 22 & a 23 & a 24 \\
a 31 & a 32 & a 33 & a 34 \\
0 & 0 & 0 & 1
\end{array}\right]
\end{aligned}
$$

where

$$
\begin{aligned}
a 11= & C b C c+C a S b \varepsilon_{z y}+S a S b S c-(C b S c+C c S a S b) \varepsilon_{x z} \varepsilon_{z y}, \\
a 12= & C a S c-S a \varepsilon_{z y}+C a C c \varepsilon_{x z} \varepsilon, \\
a 13= & C c S b-C a C b \varepsilon_{z y}-C b S a S c-(S b S c-C b C c S a) \varepsilon_{x z} \varepsilon_{z y}, \\
a 14= & y S c-x-(L+z-h) \varepsilon_{z y}+\left(y C c-\delta_{g y}+x S c+L S b S c+L C b C c S a\right) \varepsilon_{x z} \varepsilon_{z y}, \\
& +L C c S b+L C b S a S c+L C a C b \varepsilon_{z y}-\delta_{g x} \\
a 21= & C c S a S b-C a S b \varepsilon_{x z}-C b S c, \\
a 22= & S a \varepsilon_{x z}+C a C c, \\
a 23= & C a C b \varepsilon_{x z}-S b S c-C b C c S a, \\
a 24= & (L+z-h) \varepsilon_{x z}+y C c+x S c-L C a C b \varepsilon_{x z}+L S b S c+L C b C c S a-\delta_{g y}, \\
a 31= & C b C c \varepsilon_{z y}-C a S b+S a S b S c \varepsilon_{z y}+(C b S c-C c S a S b) \varepsilon_{x z}, \\
a 32= & S a+C a S c \varepsilon_{z y}-C a C c \varepsilon_{x z}, \\
a 33= & (C c S b-C b S a S c) \varepsilon_{z y}+C a C b+(S b S c+C b C c S a) \varepsilon_{x z}, \\
a 34= & L+z-h+\left(y S c-x C c-\delta_{g x}-L C c S b+L C b S a S c\right) \varepsilon_{z y} \\
& +\left(\delta_{g y}-y C c-x S c-L S b S c-L C b C c S a\right) \varepsilon_{x z}-L C a C b
\end{aligned}
$$

where $\mathbf{T}$ represents the translation matrix, $\mathbf{R}$ represents the rotation matrix. $x, y$, and $z$ represent the position coordinate value in $X$-axis, $Y$-axis, and $Z$-axis, respectively. $h$ is the workpiece width, and $a$, $b$, and $c$ represent the angle coordinate value around $A$-axis, $B$-axis, and $C$-axis, respectively, $L$ is the swing length from the $O_{t}$ to the origin of the $A$-axis coordinate system.

The proposed measurement exploits the serial orthogonal configuration of the machine tool and involves the six measurement steps shown in Fig. 9 (a)-(g). For the first measurement process, the 
machine tool is positioned at $a=b=c=0^{\circ}$, and $x=0$ as the initial position, as shown in Fig. 9 (a).

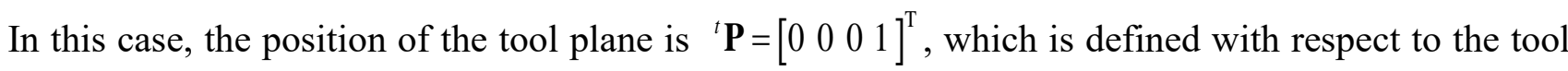
coordinate system $\left(S_{t}\left(O_{t}-X_{t} Y_{t} Z_{t}\right)\right)$. Then, the tool plane is in contact with the workpiece plane through moving the $Y$-axis and $Z$-axis, and the position of the tool plane is ${ }^{g} \mathbf{P}=\left[\begin{array}{lll}P_{g x} & P_{g y} & 0\end{array}\right]^{\mathrm{T}}$, which is defined with respect to the workpiece coordinate system $\left(S_{g}\left(O_{g}-X_{g} Y_{g} Z_{g}\right)\right)$. It is noted that the coordinate shown in the NC system at this time is $\left[\begin{array}{lll}x_{1} & y_{1} & z_{1}\end{array}\right]$. Using the Eq. (13), the equation ${ }^{g} \mathbf{P}=\mathbf{M}_{g t}{ }^{t} \mathbf{P}$ can be derived as

$$
\begin{aligned}
{\left[\begin{array}{c}
P_{g x} \\
P_{g y} \\
0 \\
1
\end{array}\right] } & =\left[\begin{array}{cccc}
1 & 0 & -\varepsilon_{z y} & -x_{1}-\left(L+z_{1}-h\right)-\delta_{g x} \\
0 & 1 & \varepsilon_{x z} & \left(L+z_{1}-h\right) \varepsilon_{x z}+y_{1}-L \varepsilon_{x z}-\delta_{g y} \\
\varepsilon_{z y} & -\varepsilon_{x z} & 1 & \left(L+z_{1}-h\right)+\left(-x_{1}-\delta_{g x}\right) \varepsilon_{z y}+\left(\delta_{g y}-y_{1}\right) \varepsilon_{x z}-L \\
0 & 0 & 0 & 1
\end{array}\right]\left[\begin{array}{l}
0 \\
0 \\
0 \\
1
\end{array}\right] \\
& =\left[\begin{array}{c}
* \\
\left(x_{1}+\delta_{g x}\right) \varepsilon_{z y}-\left(y_{1}-\delta_{g y}\right) \varepsilon_{x z}+z_{1}-h \\
1
\end{array}\right]
\end{aligned}
$$

where the symbol "*" indicates that the item is a parameter that does not need to be considered. Therefore, according to Eq.(14), the following equation is obtained:

$$
0=\left(x_{1}+\delta_{g x}\right) \varepsilon_{z y}-\left(y_{1}-\delta_{g y}\right) \varepsilon_{x z}+z_{1}-h
$$
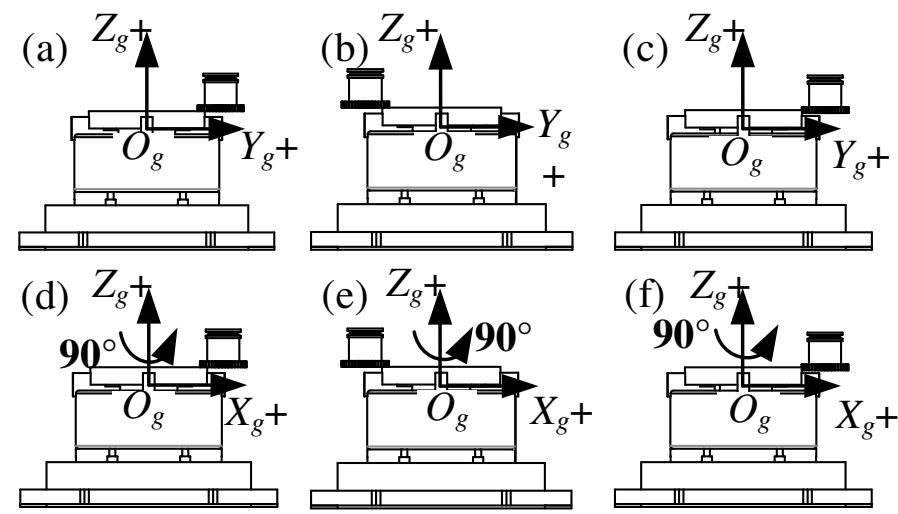

Fig. 9. The measuring method in six steps (a) the first measurement process, (b) the second measurement process, (c) the third measurement process, (d) the fourth measurement process, (e) the fifth measurement process, (f) the sixth measurement process.

For the second measurement process, the initial position is the same as the first process. However, the tool plane ${ }^{t} \mathbf{P}=\left[\begin{array}{llll}0 & 0 & 0 & 1\end{array}\right]^{\mathrm{T}}$ is in contact with the workpiece plane ${ }^{g} \mathbf{P}=\left[\begin{array}{llll}P_{g x}-P_{g y} & 0 & 1\end{array}\right]^{\mathrm{T}}$, as shown in Fig. 9 (b). In this case, the coordinate shown in the NC system is $\left[\begin{array}{lll}x_{2} & y_{2} & z_{2}\end{array}\right]$. Using the Eq. (13), the equation ${ }^{g} \mathbf{P}=\mathbf{M}_{g t}{ }^{t} \mathbf{P}$ can be derived as

$$
\left[\begin{array}{c}
P_{g x} \\
-P_{g y} \\
0 \\
1
\end{array}\right]=\left[\begin{array}{c}
* \\
* \\
\left(x_{2}+\delta_{g x}\right) \varepsilon_{z y}-\left(y_{2}-\delta_{g y}\right) \varepsilon_{x z}+z_{2}-h \\
1
\end{array}\right]
$$

Therefore, according to Eq. (16), the following equation is obtained: 


$$
0=\left(x_{2}+\delta_{g x}\right) \varepsilon_{z y}-\left(y_{2}-\delta_{g y}\right) \varepsilon_{x z}+z_{2}-h
$$

For the third measurement process, the initial position is the same as the first process. However, the tool plane ${ }^{t} \mathbf{P}=\left[\begin{array}{llll}0 & -r_{t} & 0 & 1\end{array}\right]^{\mathrm{T}}$ touches the side of the workpiece ${ }^{g} \mathbf{P}=\left[\begin{array}{llll}P_{g x} & r_{g} & P_{g z} & 1\end{array}\right]^{\mathrm{T}}$, as shown in Fig. 9 (c), where $r_{t}$ and $r_{g}$ are the radius of the tool and workpiece, respectively. In this case, the coordinate shown in the NC system is $\left[\begin{array}{lll}x_{3} & y_{3} & z_{3}\end{array}\right]$. Using the Eq. (13), the equation ${ }^{g} \mathbf{P}=\mathbf{M}_{g t}{ }^{t} \mathbf{P}$ can be derived as

$$
\left[\begin{array}{c}
P_{g x} \\
r_{g} \\
P_{g z} \\
1
\end{array}\right]=\left[\begin{array}{c}
* \\
\left(z_{3}-h\right) \varepsilon_{x z}-r_{t}-\delta_{g y}+y_{3} \\
* \\
1
\end{array}\right]
$$

Therefore, according to Eq. (18), the following equation is obtained:

$$
r_{g}=\left(z_{3}-h\right) \varepsilon_{x z}-r_{t}-\delta_{g y}+y_{3}
$$

For the fourth measurement process, the initial position is set as $a=b=0^{\circ}, c=90^{\circ}$, and $x=0$.

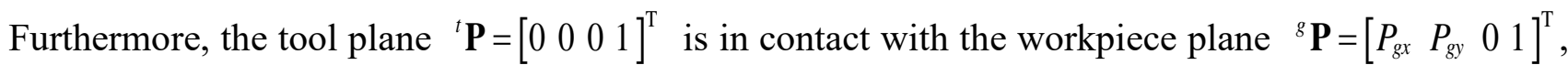
as shown in Fig. 9 (d). In this case, the coordinate shown in the NC system is $\left[\begin{array}{lll}x_{4} & y_{4} & z_{4}\end{array}\right]$. Using the Eq. (13), the equation ${ }^{g} \mathbf{P}=\mathbf{M}_{g t}{ }^{t} \mathbf{P}$ can be derived as

$$
\left[\begin{array}{c}
P_{g x} \\
P_{g y} \\
0 \\
1
\end{array}\right]=\left[\begin{array}{c}
* \\
* \\
\left(y_{4}-\delta_{g x}\right) \varepsilon_{z y}+\left(\delta_{g y}-x_{4}\right) \varepsilon_{x z}+z_{4}-h \\
1
\end{array}\right]
$$

Therefore, according to Eq.(20), the following equation is obtained:

$$
0=\left(y_{4}-\delta_{g x}\right) \varepsilon_{z y}+\left(\delta_{g y}-x_{4}\right) \varepsilon_{x z}+z_{4}-h
$$

For the fifth measurement process, the initial position is the same as the fourth process. However, the tool plane ${ }^{t} \mathbf{P}=\left[\begin{array}{llll}0 & 0 & 0 & 1\end{array}\right]^{\mathrm{T}}$ is in contact with the workpiece plane ${ }^{g} \mathbf{P}=\left[\begin{array}{llll}-P_{g x} & P_{g y} & 0 & 1\end{array}\right]^{\mathrm{T}}$, as shown in Fig. 9 (e). In this case, the coordinate shown in the NC system is $\left[\begin{array}{lll}x_{5} & y_{5} & z_{5}\end{array}\right]$. Using the Eq. (13), the equation ${ }^{g} \mathbf{P}=\mathbf{M}_{g t}{ }^{t} \mathbf{P}$ can be derived as

$$
\left[\begin{array}{c}
-P_{g x} \\
P_{g y} \\
0 \\
1
\end{array}\right]=\left[\begin{array}{c}
* \\
* \\
\left(y_{5}-\delta_{g x}\right) \varepsilon_{z y}+\left(\delta_{g y}-x_{5}\right) \varepsilon_{x z}+z_{5}-h \\
1
\end{array}\right]
$$

Therefore, according to Eq. (22), the following equation is obtained:

$$
0=\left(y_{5}-\delta_{g x}\right) \varepsilon_{z y}+\left(\delta_{g y}-x_{5}\right) \varepsilon_{x z}+z_{5}-h
$$

For the sixth measurement process, the initial position is the same as the fourth process. However,

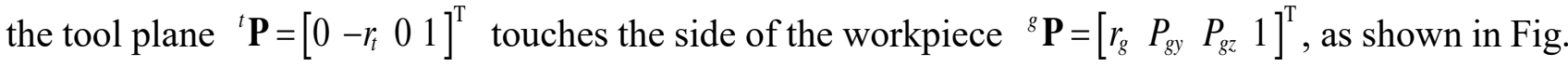
9 (f). In this case, the coordinate shown in the NC system is $\left[\begin{array}{lll}x_{6} & y_{6} & z_{6}\end{array}\right]$. Using the Eq. (13), the equation ${ }^{g} \mathbf{P}=\mathbf{M}_{g t}{ }^{t} \mathbf{P}$ can be derived as 


$$
\left[\begin{array}{c}
r_{g} \\
P_{g y} \\
P_{g z} \\
1
\end{array}\right]=\left[\begin{array}{c}
y_{6}-r_{t}-\delta_{g x}-\left(z_{6}-h\right) \varepsilon_{z y}+\left(x_{6}-\delta_{g y}\right) \varepsilon_{x z} \varepsilon_{z y} \\
* \\
* \\
1
\end{array}\right]
$$

Therefore, according to Eq. (24), the following equation is obtained:

$$
r_{g}=y_{6}-r_{t}-\delta_{g x}-\left(z_{6}-h\right) \varepsilon_{z y}+\left(x_{6}-\delta_{g y}\right) \varepsilon_{x z} \varepsilon_{z y}
$$

Solving the Eqs. (15), (17), (19), (21), (23), and (25), the main workpiece pose errors can be obtained as follows:

$$
\begin{gathered}
\varepsilon_{x z}=\frac{\left(z_{2}-z_{1}\right)-\left(x_{2}-x_{1}\right) \varepsilon_{z y}}{y_{2}-y_{1}} \\
\delta_{g y}=y_{3}-r_{t}-r_{g}+\left(z_{3}-h\right) \varepsilon_{x z} \\
\varepsilon_{z y}=\frac{\left(z_{5}-z_{4}\right)-\left(x_{5}-x_{4}\right) \varepsilon_{x z}}{y_{5}-y_{4}} \\
\delta_{g x}=y_{6}-r_{t}-r_{g}-\left(z_{6}-h\right) \varepsilon_{z y}+\left(x_{6}-\delta_{g y}\right) \varepsilon_{x z} \varepsilon_{z y}
\end{gathered}
$$

Since $\varepsilon_{x z}$ and $\varepsilon_{z y}$ are small, the $\varepsilon_{x z} \varepsilon_{z y} \approx 0$. The Eq. (29) can be represented as:

$$
\delta_{g x}=y_{6}-r_{t}-r_{g}-\left(z_{6}-h\right) \varepsilon_{z y}
$$

According to the Eqs. (26), (27), (28), (30), and the measuring position where $x=0$, it can be found that the angle deviation errors can be considered as constants, and the position deviation errors are linearly related to the angle deviation errors and the $Z$-axis coordinate. In this paper, measurement tests are performed on the gear skiving machine tool at several positions. Table 2 lists the NC system coordinate value at each step in the measurement process. Substituting the values into (26), (27), (28) and (30) can obtain the pose errors: $\delta_{g x}=-0.05 \mathrm{~mm}, \delta_{g y}=0.039 \mathrm{~mm}, \varepsilon_{z y}=-0.02^{\circ}$ and $\varepsilon_{x z}=0.013^{\circ}$, where $r_{t}=39.246 \mathrm{~mm}, r_{g}=80 \mathrm{~mm}, h=30 \mathrm{~mm}$.

Table 2 Coordinates read from control panel during the six-step measurement procedure.

\begin{tabular}{lll}
\hline $\begin{array}{l}\text { Measurement } \\
\text { item }\end{array}$ & $\begin{array}{l}\text { Preset of } A, B \text { and } \\
C \text { axis }\end{array}$ & Coordinate value $(\mathrm{mm})$ \\
\hline 1 & $\mathrm{a}=\mathrm{b}=\mathrm{c}=0^{\circ}$ & {$[0.000,80.000,31.040]$} \\
2 & $\mathrm{a}=\mathrm{b}=\mathrm{c}=0^{\circ}$ & {$[0.000,-80.000,28.960]$} \\
3 & $\mathrm{a}=\mathrm{b}=\mathrm{c}=0^{\circ}$ & {$[0.000,119.285,30.000]$} \\
4 & $a=b=0^{\circ}, c=90^{\circ}$ & {$[0.000,80.000,28.400]$} \\
5 & $a=b=0^{\circ}, c=90^{\circ}$ & {$[0.000,-80.000,31.600]$} \\
6 & $a=b=0^{\circ}, c=90^{\circ}$ & {$[0.000,119.196,30.000]$} \\
\hline
\end{tabular}

\section{Relationship between workpiece pose errors and gear skiving accuracy}

To further study the relationship between workpiece pose errors and gear skiving accuracy, the position deviation error along the $X_{g}$-axis is set as $\delta_{g x}=0.05 \mathrm{~mm}$, respectively, the position deviation error along the $Y_{g}$-axis is set as $\delta_{g y}=0.05 \mathrm{~mm}$. And the angle deviation error around $X_{g}$-axis is set as $\varepsilon_{z y}=0.05^{\circ}$, the angle deviation error around $Y_{g}$-axis is set as $\varepsilon_{x z}=0.05^{\circ}$, respectively.

\subsection{Influence of individual pose error}

In the process of gear skiving, the workpiece pose errors will cause the actual tooth flank to deviate from the theoretical tooth flank, affecting the accuracy of the skived gear. To analyze the coordinate points on any tooth flank of the skived gear, it is necessary to rotate the $i$-th enveloped tooth 
flank to the initial tooth flank $(i=1)$ in the workpiece coordinate system to evaluate the tooth deviation. The tooth deviation evaluation equation of any tooth flank can be obtained as follows:

$$
\left[\begin{array}{c}
x_{g^{\prime}} \\
y_{g^{\prime}} \\
z_{g^{\prime}} \\
1
\end{array}\right]=\left[\begin{array}{c}
x_{g}^{\left(g^{\prime}\right)} \cos \left(2 \pi \mathrm{g} \frac{i}{z_{g}}\right)+y_{g}^{\left(g^{\prime}\right)} \sin \left(2 \pi \mathrm{g} \frac{i}{z_{g}}\right) \\
-x_{g}^{\left(g^{\prime}\right)} \sin \left(2 \pi \mathrm{g} \frac{i}{z_{g}}\right)+y_{g}^{\left(g^{\prime}\right)} \cos \left(2 \pi \mathrm{g} \frac{i}{z_{g}}\right) \\
z_{g}^{\left(g^{\prime}\right)}(i) \\
1
\end{array}\right]
$$

where $\left[\begin{array}{c}x_{g}^{\left(g^{\prime}\right)} \\ y_{g}^{\left(g^{\prime}\right)} \\ z_{g}^{\left(g^{\prime}\right)} \\ 1\end{array}\right]=\mathbf{M}_{g^{\prime} g} \mathbf{M}_{g t}\left[\begin{array}{c}x_{t} \\ y_{t} \\ z_{t} \\ 1\end{array}\right], i=1,2,3, \ldots, z_{g}$.

Then, the tooth deviations including profile form deviation $f_{f \alpha}$, profile slope deviation $f_{H \alpha}$, helix form deviation $f_{f \beta}$, helix slope deviation $f_{H \beta}$, single flank deviation $f_{p t}$, and cumulative pitch deviation $F_{p}$ can be derived by

$$
\Delta\left(f_{H \alpha}, f_{f \alpha}, f_{H \beta}, f_{f \beta}, f_{p t}, F_{p}\right)=r_{g}^{\prime}-r_{g}
$$

where $r_{g}^{\prime}$ is actual tooth flank $r_{g}$ is the theoretical tooth flank.

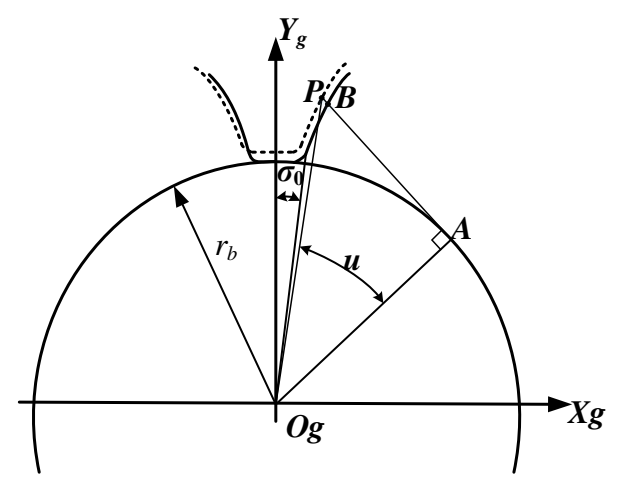

Fig. 10. Evaluation of tooth profile deviation.

According to Fang et al. [25], in order to analyze the sensitivity of gear skiving accuracy to the workpiece pose errors, $\Delta\left(f_{H \alpha}, f_{f \alpha}, f_{H \beta}, f_{f \beta}, f_{p t}, F_{p}\right)$ are calculated in the transverse section, as shown in Fig. 10. The normal distance $|P B|$ from any point $P$ on the actual tooth flank to the theoretical tooth flank represents the tooth deviation. Therefore, the normal evaluation equation of any point on the actual tooth flank can be obtained

$$
|P B|=|P A|-|A B|
$$

where $|P A|=\sqrt{x_{g^{\prime}}^{2}+y_{g^{\prime}}^{2}-r_{b}^{2}}, u=\left|a \tan \left(\frac{y_{g^{\prime}}}{x_{g^{\prime}}}\right)\right|+a \tan \left(\frac{|P A|}{r_{b}}\right)-\sigma_{0},|A B|=r_{b} u, x_{g^{\prime}}$ and $y_{g^{\prime}}$ is the coordinate of the point $P, r_{b}$ is the base circle radius of the workpiece, $\sigma_{0}$ is half of the angular tooth thickness.

According to the Eqs. (32) and (33), the profile deviation between the actual tooth flank and theoretical tooth flank on the transverse section can be calculated. Then the profile deviation, helix deviation, and pitch deviation can be calculated and evaluated based on ISO 1328:2013 [26]. The numerical analyses are performed to study the influence of individual workpiece pose errors on gear 
skiving accuracy by the basic parameters listed in Table 3 . The positive deviation is taken as a positive value and the negative deviation as a negative value.

Table 3 Basic parameters of gear skiving in analysis.

\begin{tabular}{llll}
\hline Items & Value & Items & Value \\
\hline Tooth number of tool $\left(z_{t}\right)$ & 35 & Tooth number of workpiece $\left(z_{g}\right)$ & 70 \\
Helix angle of tool $\left(\beta_{t}\right)$ & $20^{\circ}$ & Helix angle of workpiece $\left(\beta_{g}\right)$ & $0^{\circ}$ \\
Normal module $\left(m_{n}\right)$ & $2 \mathrm{~mm}$ & Workpiece width $(h)$ & $30 \mathrm{~mm}$ \\
Normal pressure angle $\left(\alpha_{n}\right)$ & $20^{\circ}$ & Shaft angle $(\Sigma)$ & $20^{\circ}$ \\
Radius of tool $\left(r_{t}\right)$ & $39.246 \mathrm{~mm}$ & Center distance $(a)$ & $32.754 \mathrm{~mm}$ \\
\hline
\end{tabular}
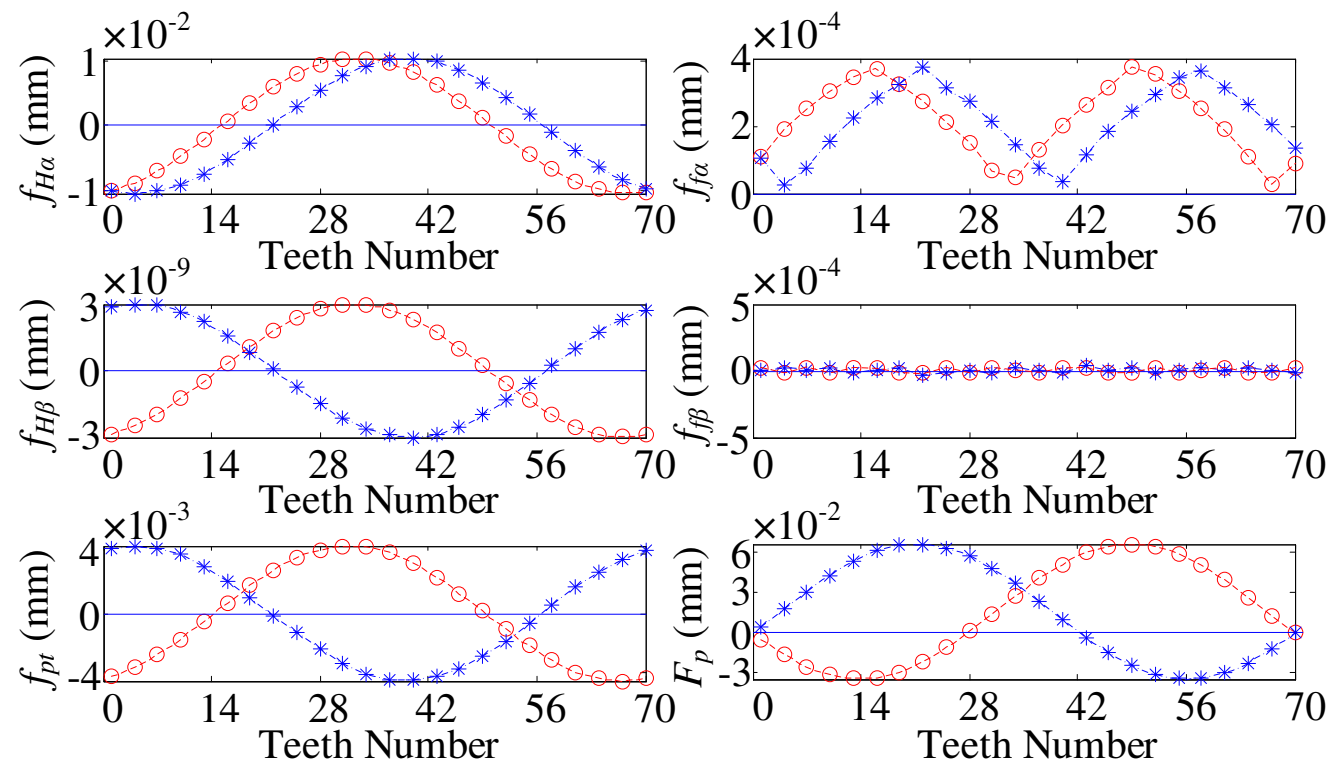

$--\ominus-$ Left Flank

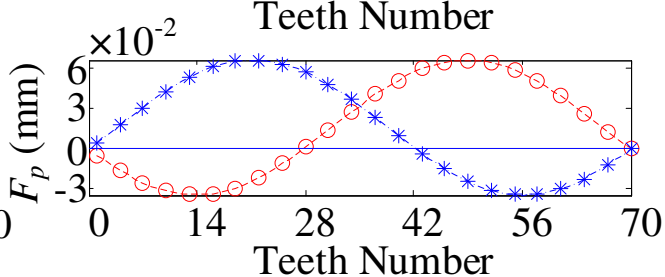

-*- Right Flank

Fig. 11. Influence of individual workpiece position deviation error $\left(\delta_{g x}=0.05 \mathrm{~mm}\right)$ on tooth deviation.

As shown in Fig. 11 to Fig. 12, the influences of individual workpiece position deviation error on the tooth deviation are analyzed. In the gear accuracy evaluation, the maximum error on the right and left flank are selected as the evaluation value of tooth deviation. If the absolute value of maximum tooth deviation is less than $5 \mu \mathrm{m}$, it will be considered as an insensitive error with respect to pose error. Otherwise, it is considered as a sensitive error. It can be found that tooth deviation evaluated from the maximum error of each tooth flank presents regular cosine or sine curves. With the change of the phase angle of the position deviation error, the number of teeth corresponding to the maximum tooth deviation is also changing. In Fig. 11 to Fig. 12, the maximum tooth deviation of $f_{f \alpha}, f_{f \beta}, f_{H \beta}$, and $f_{p t}$ is less than $5 \mu \mathrm{m}$, so it can be considered that these tooth deviations are insensitive to workpiece position deviation errors. However, the maximum tooth deviation of $f_{H \alpha}$ and $F_{p}$ is more than $5 \mu \mathrm{m}$, so they are sensitive to workpiece position deviation errors. 

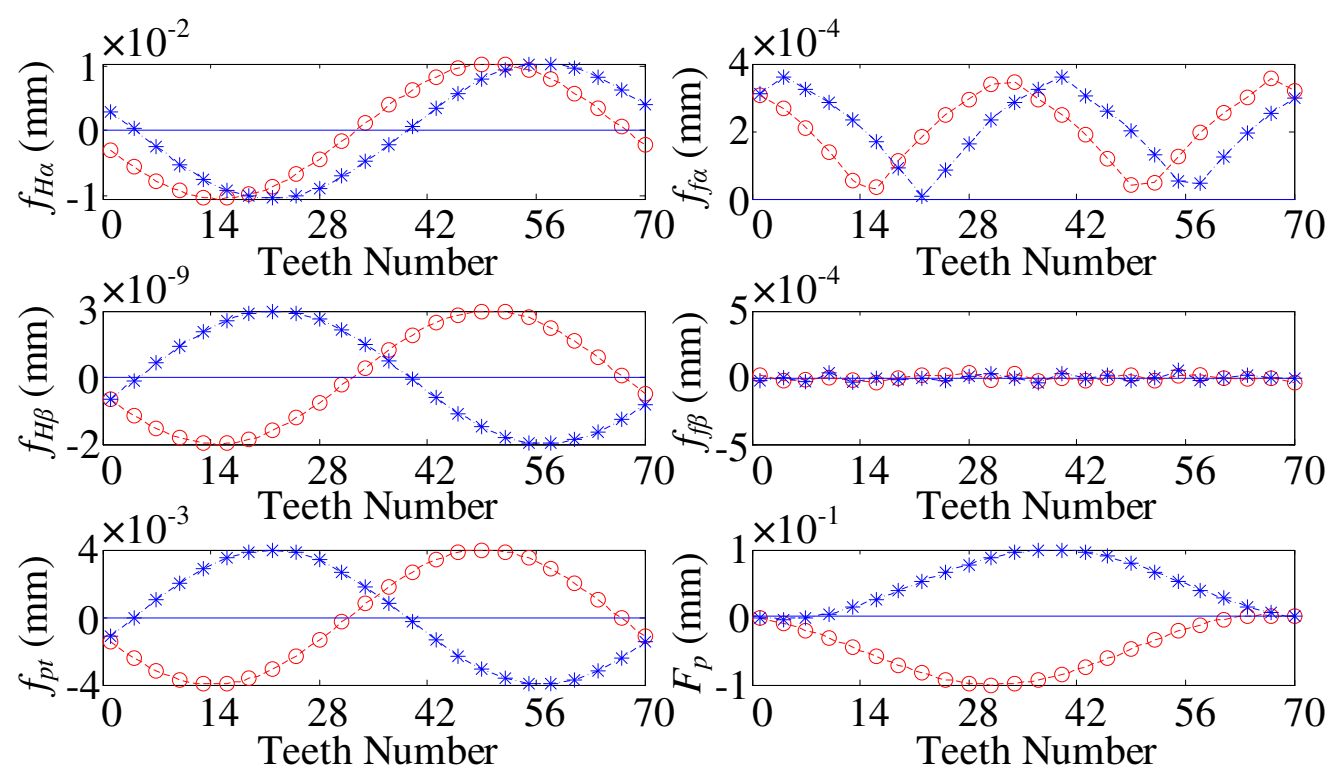

Left Flank

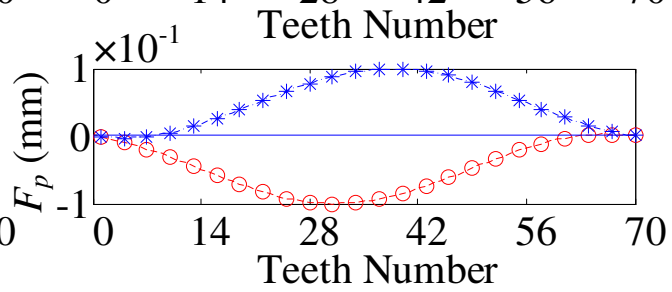

\section{-- *- Right Flank}

Fig. 12. Influence of individual workpiece position deviation error $\left(\delta_{g y}=0.05 \mathrm{~mm}\right)$ on tooth deviation.

As shown in Fig. 13 to Fig. 14, the influences of individual workpiece angle deviation error on the tooth deviation are analyzed. The tooth deviation curves also show regular cosine or sine curves, and the curves also change with the phase angle of angle deviation error. It can be found that $f_{H \alpha}$, $f_{f \alpha}, f_{f \beta}$, and $f_{p t}$ are insensitive to workpiece angle deviation errors. However, $f_{H \beta}$ and $F_{p}$ are sensitive to workpiece angle deviation errors.
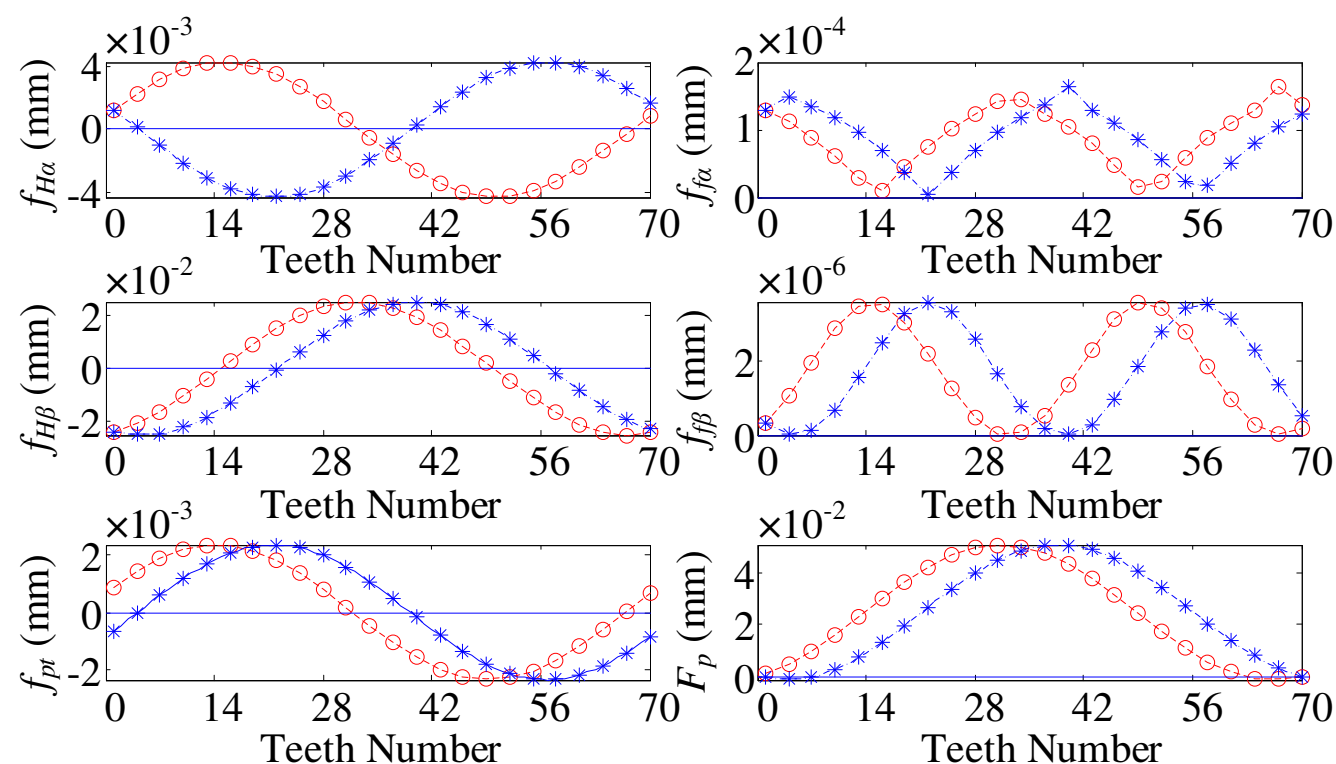

Left Flank

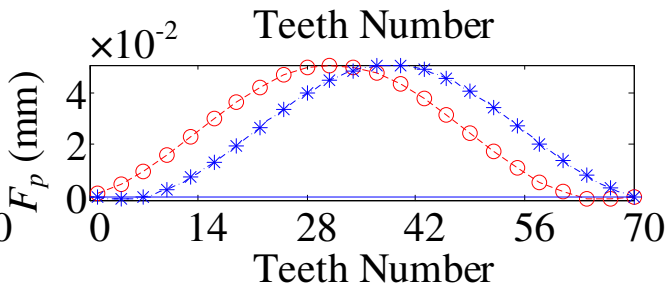

*- Right Flank

Fig. 13. Influence of individual workpiece angle deviation error $\left(\varepsilon_{z y}=0.05^{\circ}\right)$ on tooth deviation. 

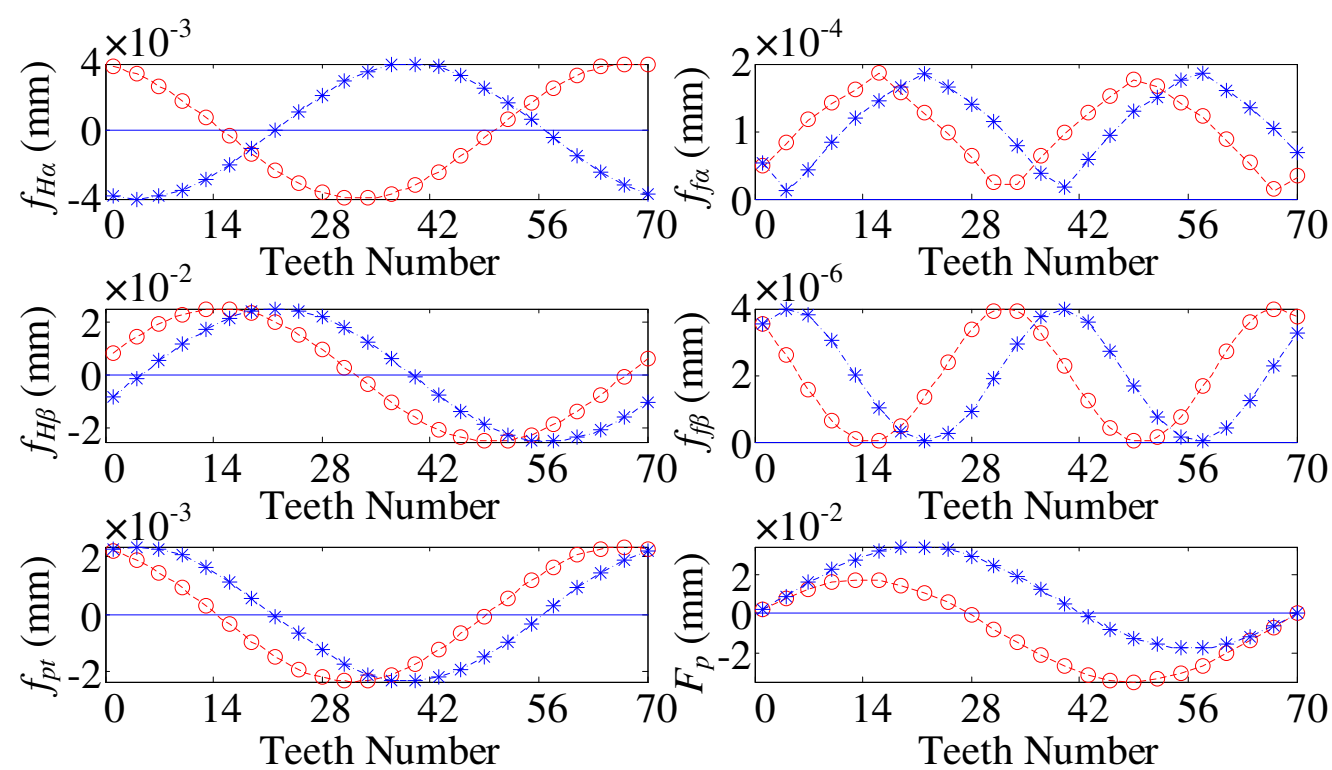

Teeth Number - Left Flank

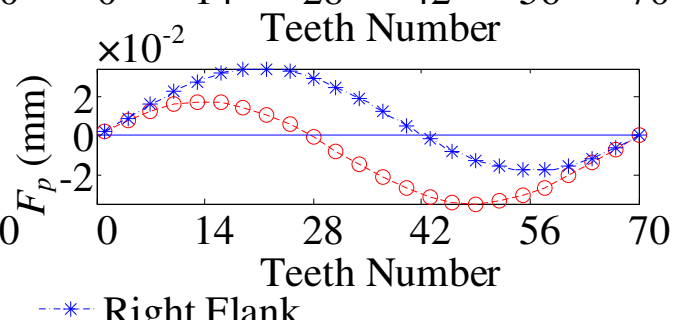

*- Right Flank

Fig. 14. Influence of individual workpiece angle deviation error $\left(\varepsilon_{x z}=0.05^{\circ}\right)$ on tooth deviation.

Table 4 The numerical results of individual workpiece pose error on the right flank $(\mu \mathrm{m})$.

\begin{tabular}{ccccccc}
\hline & \multicolumn{2}{c}{$f_{\alpha}$} & \multicolumn{2}{c}{$f_{\beta}$} & \multicolumn{2}{c}{$F_{p}\left( \pm f_{p t}\right)$} \\
\cline { 2 - 7 } Pose errors $(\mathrm{mm})$ & $f_{H \alpha}$ & $f_{f \alpha}$ & $f_{H \beta}$ & $f_{f \beta}$ & $f_{p t}$ & $F_{p}$ \\
\hline$\delta_{g x}=0.05, \delta_{g y}=0, \varepsilon_{z y}=0^{\circ}, \varepsilon_{x z}=0^{\circ}$ & $\mathbf{1 1}$ & $<1$ & $<1$ & $<1$ & $<5$ & $\mathbf{6 6}$ \\
$\delta_{g x}=0, \delta_{g y}=0.05, \varepsilon_{z y}=0^{\circ}, \varepsilon_{x z}=0^{\circ}$ & $\mathbf{1 0}$ & $<1$ & $<1$ & $<1$ & $<5$ & $\mathbf{9 7}$ \\
$\delta_{g x}=0, \delta_{g y}=0, \varepsilon_{z y}=0.05^{\circ}, \varepsilon_{x z}=0^{\circ}$ & $<5$ & $<1$ & $\mathbf{2 5}$ & $<1$ & $<3$ & $\mathbf{5 1}$ \\
$\delta_{g x}=0, \delta_{g y}=0, \varepsilon_{z y}=0^{\circ}, \varepsilon_{x z}=0.05^{\circ}$ & $<5$ & $<1$ & $\mathbf{2 5}$ & $<3$ & $<3$ & $\mathbf{3 7}$ \\
\hline
\end{tabular}

Table 4 shows the maximum tooth deviation on the right flank. In summary, the profile slope deviation $f_{H \alpha}$ and cumulative pitch deviation $F_{p}$ are sensitive to workpiece position deviation errors. The helix slope deviation $f_{H \beta}$ and cumulative pitch deviation $F_{p}$ are sensitive to workpiece angle deviation errors. The diversity of the tooth deviation on both flanks is determined by the phase angle of workpiece pose errors. The conclusions can be used to determine the existence of pose errors and the phase angle of the pose errors.

\subsection{Relationship between individual pose error and coupling pose errors}

In this section, the pose errors are applied together to study the coupling effect. In Fig. 15, the influence of the coupling pose errors on tooth deviation is analyzed. The tooth deviation curves show regular cosine or sine curves, and the analyzed results show that the $f_{H \alpha}, f_{H \beta}, f_{p t}$, and $F_{p}$ are sensitive to coupling pose errors. The maximum deviation values are $22 \mu \mathrm{m}, 36 \mu \mathrm{m}, 9 \mu \mathrm{m}$, and $204 \mu \mathrm{m}$, respectively. 

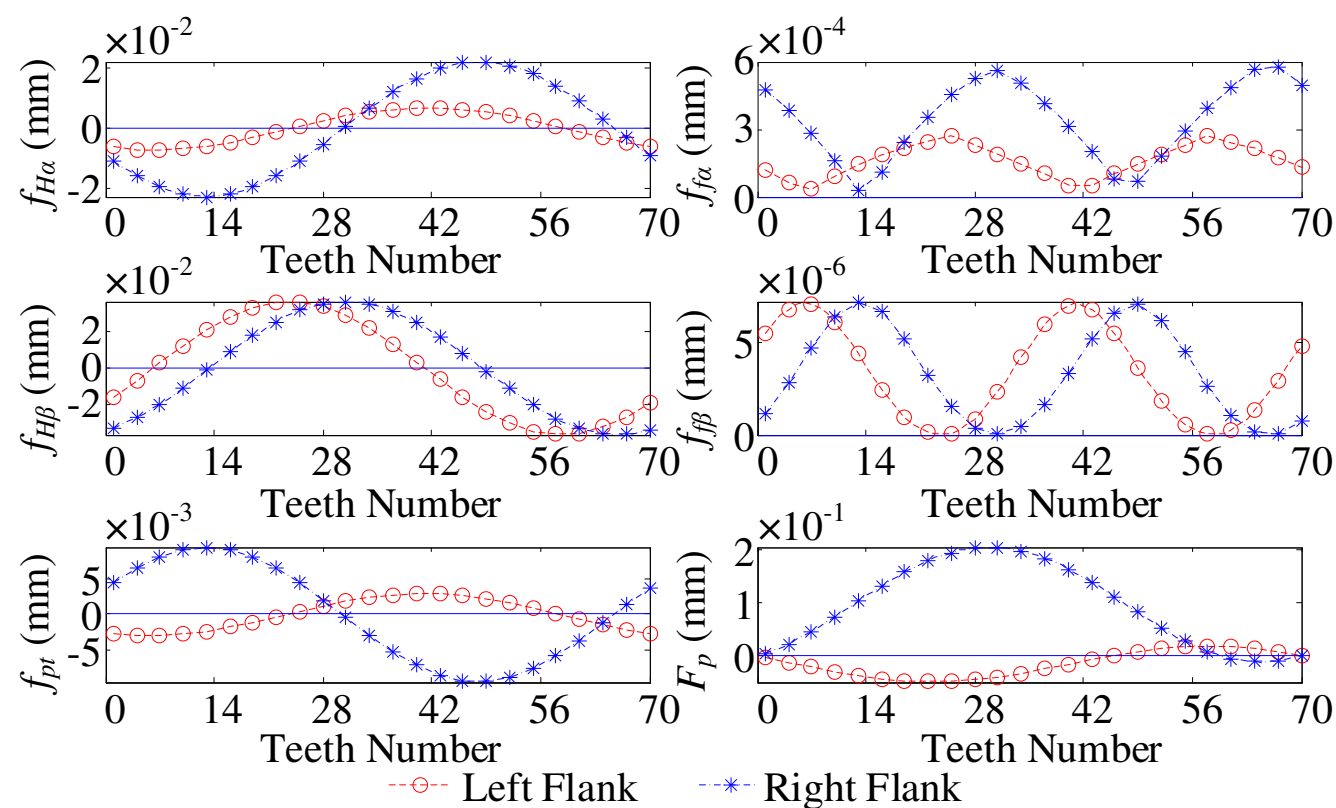

Fig. 15. Influence of the coupling pose errors $\left(\delta_{g x}=0.05 \mathrm{~mm}, \delta_{g y}=0.05 \mathrm{~mm}, \varepsilon_{z y}=0.05^{\circ}, \varepsilon_{x z}=0.05^{\circ}\right)$ on tooth deviation.

From the above analysis, it can be concluded that the workpiece pose errors mainly affect the profile slope deviation $f_{H \alpha}$, helix slope deviation $f_{H \beta}$, and cumulative pitch deviation $F_{p}$. Therefore, this paper mainly focuses on studying the relationship between individual pose error and coupling pose errors on these three deviations.

For simplicity, $f^{C}\left(f_{H \alpha}, f_{H \beta}, F_{p}\right)$ is used to represent the influence of the coupling pose errors on tooth deviation, $f^{I}\left(f_{H \alpha}, f_{H \beta}, F_{p}\right)$ is used to represent the influence of individual pose error on tooth deviation, and $\sum f^{I}\left(f_{H \alpha}, f_{H \beta}, F_{p}\right)$ is used to represent the linear superposition of influence of individual error on the deviation. According to [27,28], the influences of the coupling errors on deviation are regarded as the linear superposition of influence of individual error on deviation under different errors when machining bevel gear and grinding gear. Therefore, assumed that $f^{C}\left(f_{H \alpha}, f_{H \beta}, F_{p}\right)$ is also a linear superposition of each $f^{I}\left(f_{H \alpha}, f_{H \beta}, F_{p}\right)$ in gear skiving system, and then calculate the difference between the $f^{C}\left(f_{H \alpha}, f_{H \beta}, F_{p}\right)$ and $\sum f^{I}\left(f_{H \alpha}, f_{H \beta}, F_{p}\right)$, the following equations can be obtained

$$
\begin{gathered}
{\left[\begin{array}{c}
f_{H \alpha}^{C} \\
f_{H \beta}^{C} \\
F_{P}^{C}
\end{array}\right]=\left[\begin{array}{lll}
k_{1} & k_{2} & k_{3}
\end{array}\right]\left[\begin{array}{c}
\sum f_{H \alpha}^{I} \\
\sum f_{H \beta}^{I} \\
\sum F_{P}^{I}
\end{array}\right]+\left[\begin{array}{c}
\Delta f_{H \alpha} \\
\Delta f_{H \beta} \\
\Delta F_{P}
\end{array}\right]} \\
{\left[\begin{array}{c}
\Delta f_{H \alpha} \\
\Delta f_{H \beta} \\
\Delta F_{P}
\end{array}\right]=\left[\begin{array}{c}
f_{H \alpha}^{C} \\
f_{H \beta}^{C} \\
F_{P}^{C}
\end{array}\right]-\left[\begin{array}{c}
\sum f_{H \alpha}^{I} \\
\sum f_{H \beta}^{I} \\
\sum F_{P}^{I}
\end{array}\right]}
\end{gathered}
$$

where superscripts $C$ and $I$ are coupling pose errors and individual pose error, respectively, and $k_{i}(i=1,2,3)$ is the linear correlation coefficient.

According to Eqs. (34) and (35), the numerical results can be obtained in Fig. 16 and Fig. 17. With the increase of the index of tooth number, both position deviation errors and angle deviation errors contribute to the maximum tooth deviation, the curves of $f^{C}\left(f_{H \alpha}, f_{H \beta}, F_{p}\right)$ is consistent with the curves of $\sum f^{I}\left(f_{H \alpha}, f_{H \beta}, F_{p}\right)$, as shown in Fig. 16 and Fig. 17, the difference $\Delta_{i}\left(i=f_{H \alpha}, f_{H \beta}, F_{P}\right)$ 
between the $f^{C}\left(f_{H \alpha}, f_{H \beta}, F_{p}\right)$ and $\sum f^{I}\left(f_{H \alpha}, f_{H \beta}, F_{p}\right)$ is very small, and the coefficient $k_{i}(i=1,2,3)$ is basically equal to 1 . Therefore, the influences of the coupling errors on deviation can be approximately regarded as the linear superposition of the influences of the individual error on deviation under different errors in the gear skiving process. This rule is also consistent with the regulations of the cutting depth $\Delta d$ in Section 3.2. Moreover, this method can also determine whether position errors or angle deviation errors and whether each pose error has the same phase. It is beneficial to trace and compensate for the pose error of the gear skiving process.

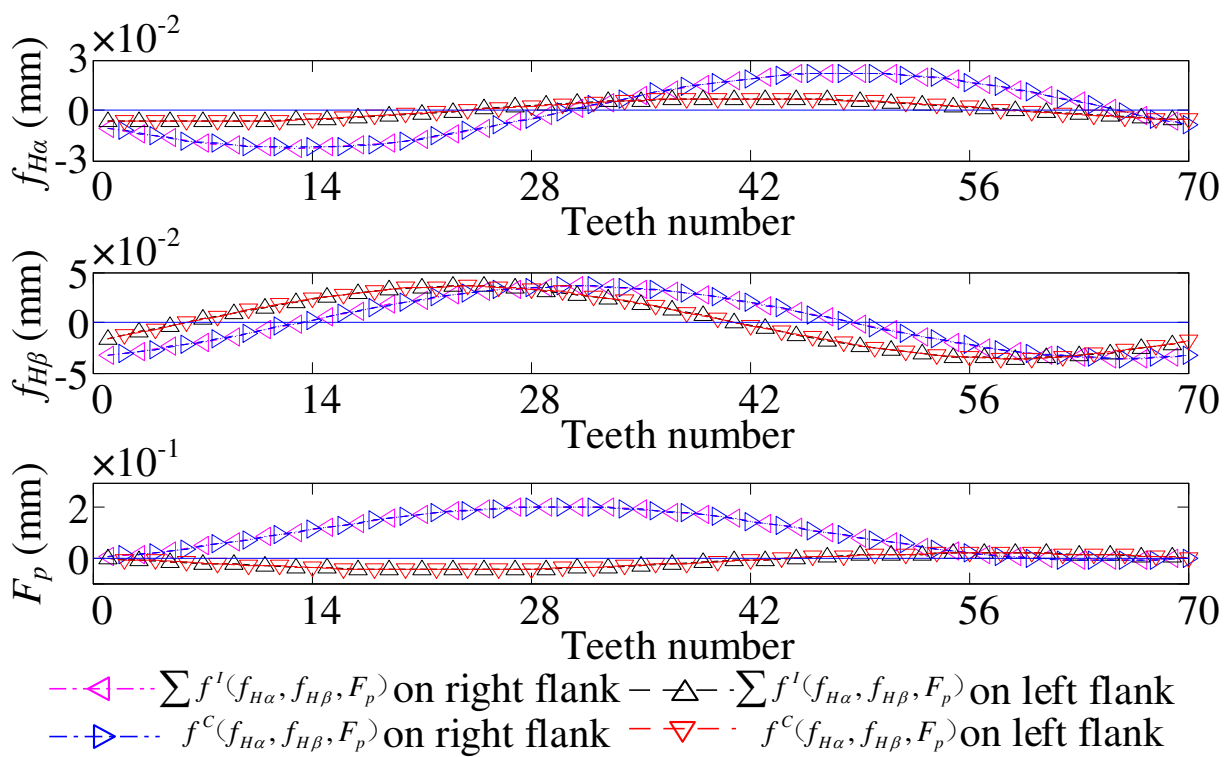

Fig. 16. The relationship between coupling errors and linear superposition with respect to individual tooth deviation of different errors.
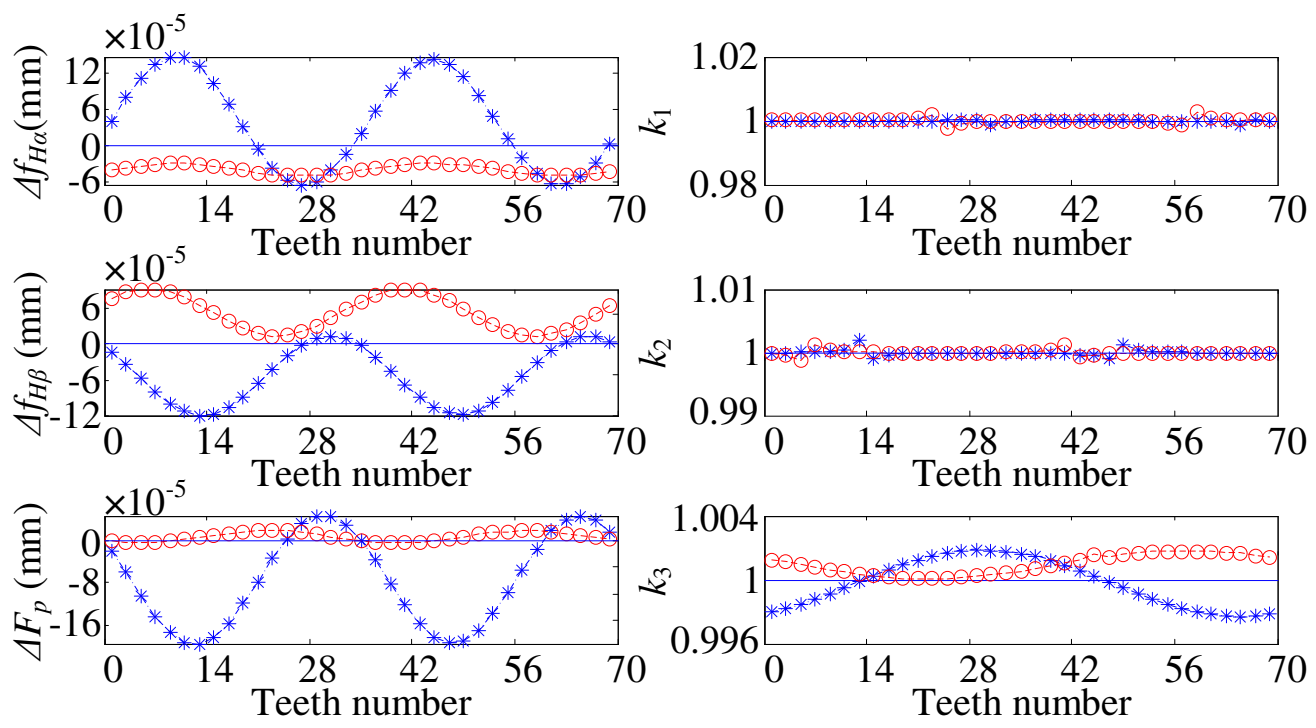

Left Flank

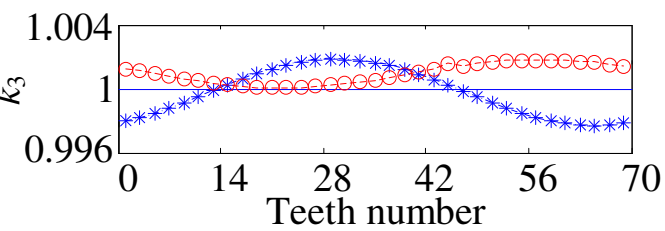

Right Flank

Fig. 17. The corresponding coefficient between coupling errors and linear superposition of individual error.

\section{Experiment and analysis}




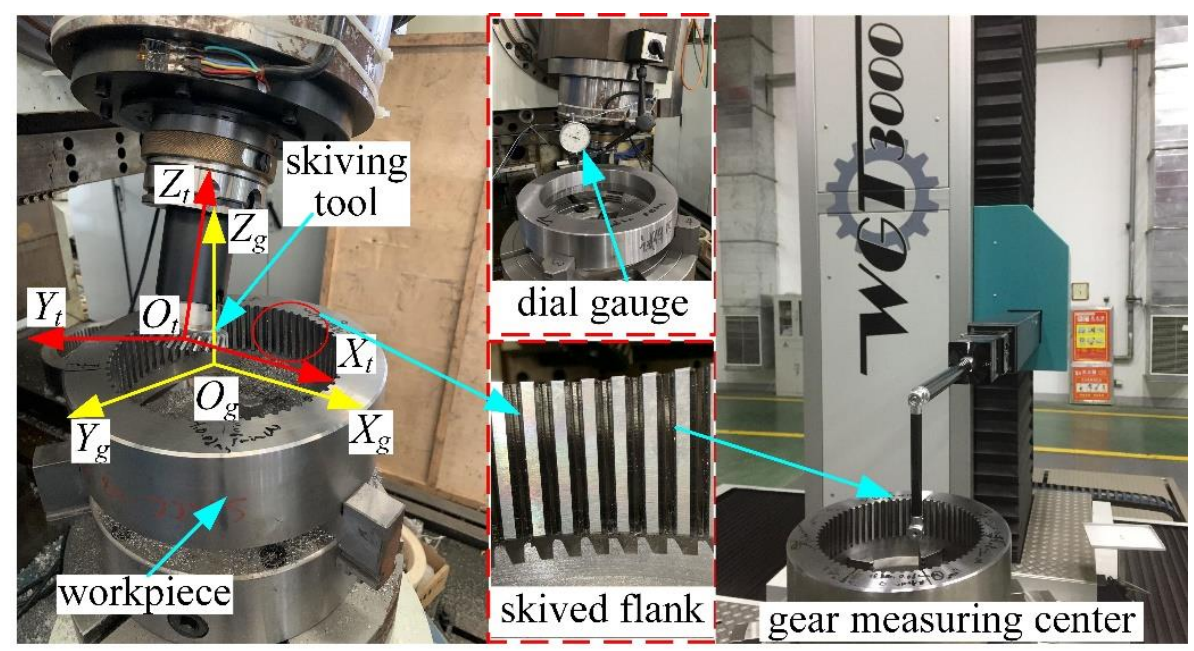

Fig. 18. Actual gear skiving experiment and measurement.

As shown in Fig. 18, three typical machining workpiece pose errors are selected to process on the gear skiving machine tool to verify the proposed numerical analysis method. In order to reduce the influence of other factors, it is necessary to control the process of the experiment. The geometric accuracy of the gear skiving machine tool is checked and compensated to reduce the influence of irrelevant errors. The machine tool is idling for 2 hours before gear skiving, reducing the influence of thermal error. Moreover, the machining parameters used in each group of experiments are set to the same so that the main factor that affects the gear skiving accuracy in the machining process is the workpiece pose errors.

According to the setting value in Table 5, first of all, the workpiece pose errors can be adjusted manually by a dial gauge. Then the proposed measurement method in Section 4 is used to measure the workpiece pose errors to further verify whether the error value in the experiments meets the requirements. The basic parameters of the gear skiving used in the experiment are consistent with Table 3. After skiving, the teeth of the workpiece are sorted sequentially, number 1, 23, and 47 are selected to measure in gear measuring center, as shown in Fig. 18.

Table 5 Workpiece pose error setting value for different conditions.

\begin{tabular}{cc} 
Test number & Pose error setting value \\
\hline 1 & $\delta_{g x}=0.05, \delta_{g y}=0, \varepsilon_{z y}=0^{\circ}, \varepsilon_{x z}=0^{\circ}$ \\
2 & $\delta_{g x}=0, \delta_{g y}=0, \varepsilon_{z y}=0.05^{\circ}, \varepsilon_{x z}=0^{\circ}$ \\
3 & $\delta_{g x}=0.05, \delta_{g y}=0.05, \varepsilon_{z y}=0.05^{\circ}, \varepsilon_{x z}=0.05^{\circ}$ \\
\hline
\end{tabular}

According to the analysis result in Section 5, the profile slope deviation $f_{H \alpha}$, helix slope deviation $f_{H \beta}$, cumulative pitch deviation $F_{p}$ are sensitive to the workpiece pose errors. Therefore, these deviations are analyzed and measured. Table 6 shows the comparison between numerical results and experiment results on the selected right tooth flanks. Since the on-site processing environment may affect the gear skiving, the actual measurement results are slightly larger than the numerical analysis results. However, the overall trend of the measurement accuracy is consistent with the numerical results. According to Table 6, in actual skiving, $f_{H \alpha}$ is sensitive to position deviation errors, $f_{H \beta}$ is sensitive to angle deviation errors, and $F_{p}$ is sensitive to position deviation errors and angle deviation errors. The experimental results verify the numerical simulation results. Therefore, this method can be used to trace the position and angle deviation errors of the workpiece and guide the setting requirements of the workpiece in gear skiving. 
Table 6 Comparison of numerical results and experimental results $(\mu \mathrm{m})$.

\begin{tabular}{cccccccc}
\hline \multirow{2}{*}{ Test number } & \multirow{2}{*}{ Deviation items } & \multicolumn{3}{c}{ Numerical results } & \multicolumn{4}{c}{ Experimental results } \\
\cline { 2 - 8 } & & 1 & 23 & 47 & 1 & 23 & 47 \\
\hline \multirow{3}{*}{1} & $f_{H \alpha}$ & $\mathbf{- 9 . 9}$ & $\mathbf{1 . 1}$ & $\mathbf{8}$ & $\mathbf{- 1 0 . 6}$ & $\mathbf{1 . 5}$ & $\mathbf{1 0 . 3}$ \\
& $f_{H \beta}$ & - & - & - & 0.7 & -0.1 & -0.2 \\
& $F_{p}$ & $\mathbf{4 . 3}$ & $\mathbf{6 5 . 4}$ & $\mathbf{1 7 . 9}$ & $\mathbf{6}$ & $\mathbf{7 0 . 6}$ & $\mathbf{2 0 . 2}$ \\
2 & $f_{H \alpha}$ & - & - & - & 3.5 & -4.9 & 2 \\
& $f_{H \beta}$ & $\mathbf{- 2 3 . 9}$ & $\mathbf{1 . 9}$ & $\mathbf{2 0}$ & $\mathbf{- 2 3 . 3}$ & $\mathbf{2 . 5}$ & $\mathbf{1 9 . 7}$ \\
& $F_{p}$ & - & $\mathbf{2 9}$ & $\mathbf{4 4}$ & $\mathbf{2 . 5}$ & $\mathbf{3 1 . 2}$ & $\mathbf{4 6 . 3}$ \\
3 & $f_{H \alpha}$ & $\mathbf{- 1 1}$ & $\mathbf{- 1 4}$ & $\mathbf{2 2 . 1}$ & $\mathbf{- 1 2 . 5}$ & $\mathbf{- 1 5 . 6}$ & $\mathbf{3 6 . 7}$ \\
3 & $f_{H \beta}$ & $\mathbf{- 3 2 . 1}$ & $\mathbf{2 7 . 2}$ & $\mathbf{4 . 6}$ & $\mathbf{3 3 . 3}$ & $\mathbf{2 8 . 2}$ & $\mathbf{5 . 9}$ \\
& $F_{p}$ & $\mathbf{4 . 6}$ & $\mathbf{1 8 4 . 4}$ & $\mathbf{1 0 0 . 9}$ & $\mathbf{5 . 0}$ & $\mathbf{2 0 3 . 3}$ & $\mathbf{1 1 2 . 4}$ \\
\hline
\end{tabular}

In this study, the PSO algorithm is used to further study the relationship between the workpiece pose errors and the gear skiving accuracy to assist the proposed method to find the maximum allowable workpiece pose errors in gear skiving under different conditions.

In the actual gear skiving process, most of the processing gear module affected by the existing technology is small, and the maximum diameter of the workpiece that can be skived is also limited. Therefore, in two cases, the optimal global prediction of the workpiece pose errors is based on the PSO algorithm: one of the modules is 2 , the tooth number of the workpiece ranges is $60-120$, and the other of the tooth number is 70 , the module range is $0.5-3$.

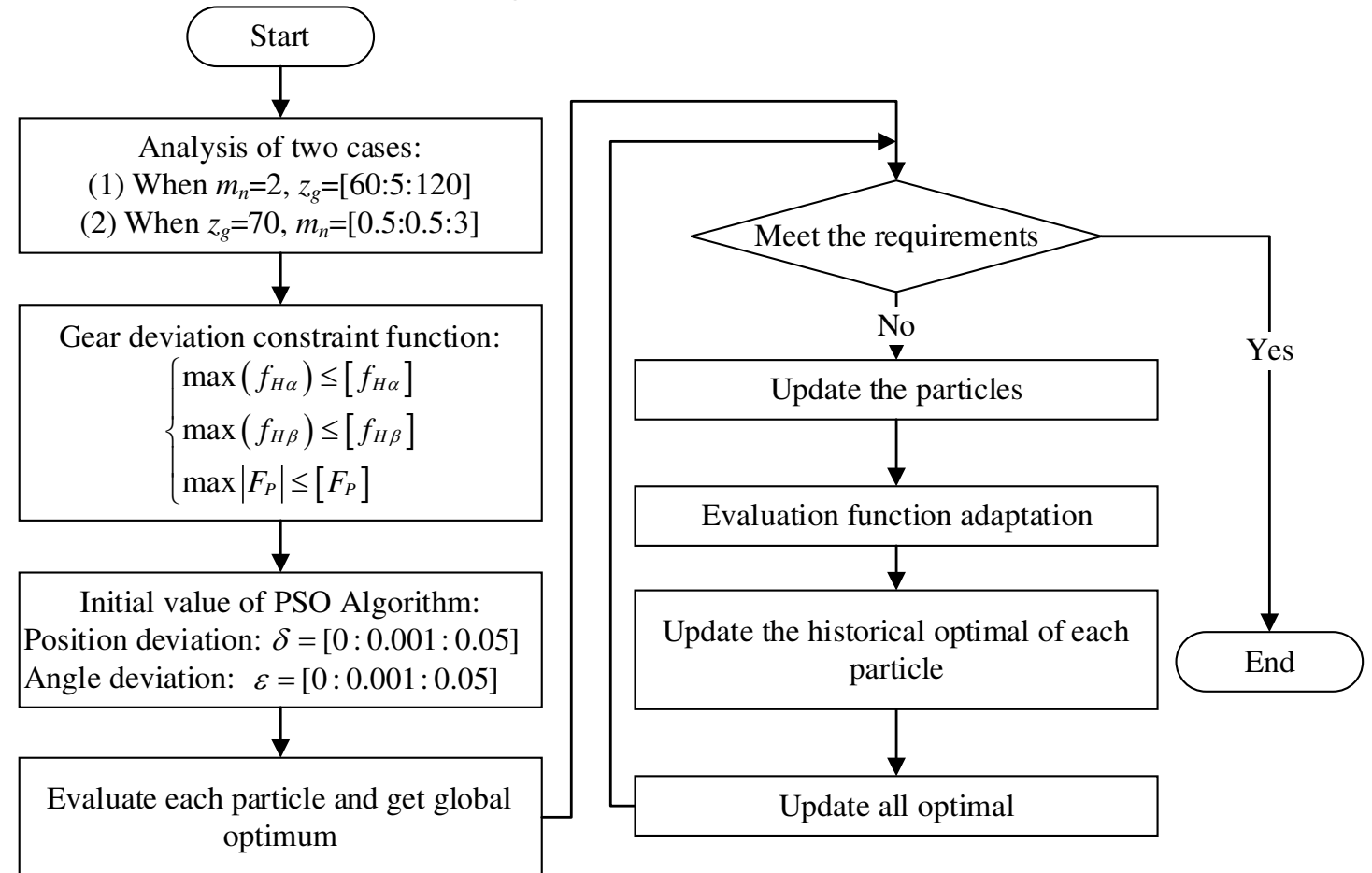

Fig. 19 Workpiece pose errors prediction based on PSO algorithm.

After previous analysis, it shows that $f_{H \alpha}, f_{H \beta}$, and $F_{p}$ are more sensitive to the workpiece pose errors. Therefore, we consider these three deviations as the target. To reduce the calculation amount, the position deviation errors and the angle deviation errors in one direction are considered, respectively, which is also more in line with the actual machining situation. Then the individual position deviation error and angle deviation error are randomly set as the initial value of the PSO 
algorithm. Through the evaluation of calculation accuracy, the required workpiece pose errors are finally obtained. The detailed process of the prediction is shown in Fig. 19.

In this paper, the machining accuracy in ISO 5 [26] is taken as the optimization target, and the prediction results are shown in Fig. 20. In Fig. 20 (a), if the number of the module does not change, the position deviation error increases with the increase of the tooth number. In contrast, the angle deviation error remains basically unchanged. If the number of gears does not change, the position deviation error increases step by step with the increase of module, while the angle deviation error remains unchanged, the detail is described in Fig. 20 (b).
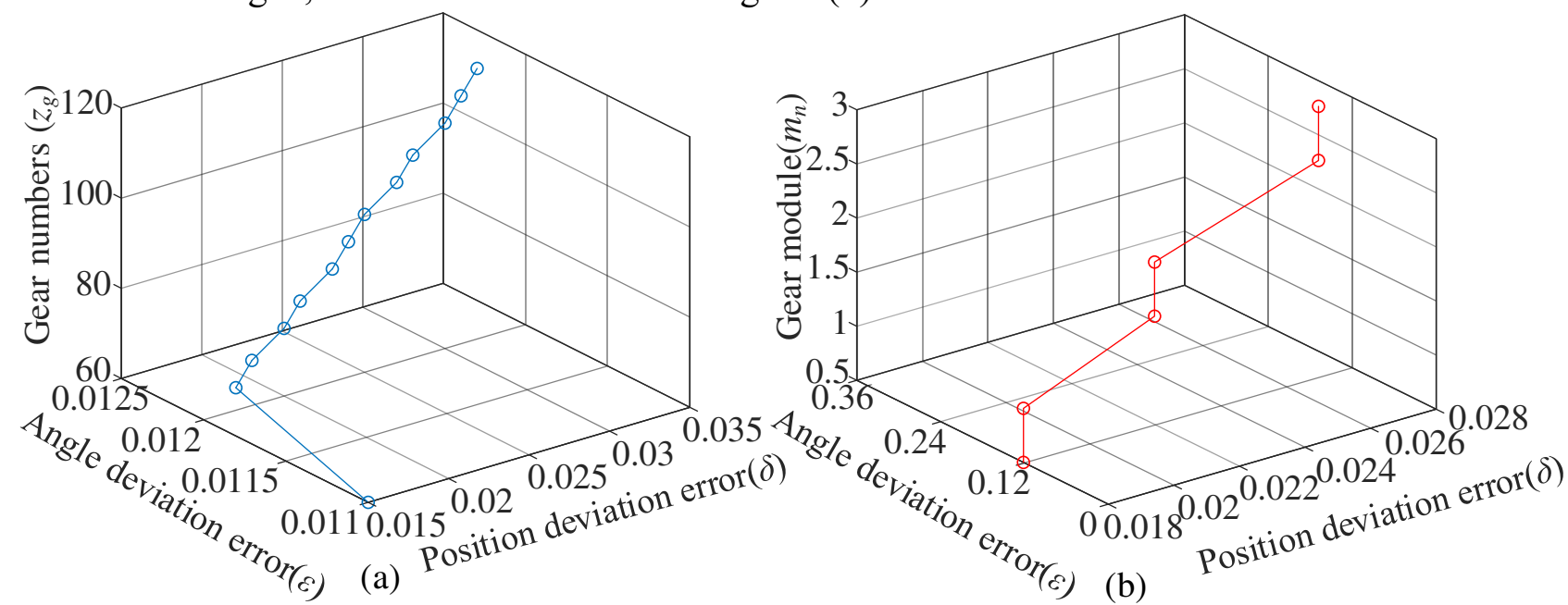

\section{Fig. 20. The workpiece pose errors. (a) Different gear numbers when $m_{n}=2$. (b) Different gear module when $z_{g}=70$.}

Therefore, it can be known that the control of angle deviation error is relatively strict in the skiving process, which is conducive to ensure that the helix slope deviation $f_{H \beta}$ is within the allowable range. In contrast, the acceptable range of the position deviation error can be appropriately increased with an increase in the number of gears or modules. This is because as the number of gears or modules increases, the diameter of the gear also increases so that the position deviation error will have a relatively small impact on the accuracy of the gear skiving. The actual processing has verified this problem to obtain the same results. Therefore, the method mentioned in this paper can be used to guide and quickly trace the source of the workpiece pose errors in the actual gear skiving process.

\section{Conclusions}

This paper studied the influences of workpiece pose errors on gear skiving accuracy by numerical simulation and experimental analysis. Several conclusions can be reached as follows:

(1) The workpiece pose errors of the gear skiving system are defined and analyzed, and the main error items in the setting process are identified. The regulations between each pose error and cutting depth are investigated. A measurement method of workpiece pose errors based on actual inverse kinematics is proposed, and the measurement of the pose errors is realized through six steps.

(2) The numerical simulation results show that the profile slope deviation $f_{H \alpha}$ and helix slope deviation $f_{H \beta}$ are sensitive to position and angle deviation errors, respectively. The cumulative pitch deviation $F_{p}$ is sensitive to both position and angle deviation errors. The results can be used to trace the workpiece pose errors.

(3) The influence of the coupling pose errors on deviation can be approximately regarded as a linear superposition of the influence of individual pose errors on deviation under different errors in the gear skiving process.

(4) The numerical analysis results are verified by actual gear skiving. The PSO algorithm is 
introduced to study the relationship between the workpiece pose errors and the number of gears and modules. With the change of module and the number of gears, the angle deviation error is almost unaffected. In contrast, the position deviation error increases almost linearly with the increase of the number of gears and increases step by step with the increase of module.

\section{Author contribution}

Xiaochuan Lin: Conceptualization, Methodology, Software, Validation, Writing - Original Draft. Rongjing Hong: Supervision, Funding acquisition, Writing - Review \& Editing. Yawen Wang: Writing - Review \& Editing, Software. Yun Peng: Validation. Xiaolei Ren: Writing - Review \& Editing.

\section{Funding}

This work was supported by the National Natural Science Foundation of China (Grant No. 51635003) and the Postgraduate Research \& Practice Innovation Program of Jiangsu Province (Grant No.KYCX18_1092).

\section{Data availability}

The authors confirm that the data supporting the findings of this study are available within the article.

\section{Compliance with ethical standards}

Competing interests The authors declare that they have no competing interests.

Ethical approval Not applicable.

Consent to participate Not applicable.

Consent for publish Not applicable.

\section{References}

1. MONDEN T, KIKUCHI T, CHIHARA Y, NAKAMURA Y (2015) MHI Super-Skiving System for Longer Tool Life and Enhanced Efficiency in Internal Gear Cutting. Mitsubishi Heavy Ind Tech Rev 52:101-105

2. Li J, Chen X, Zhang H (2011) Slicing technology for cylindrical gears. Jixie Gongcheng Xuebao/Journal Mech Eng 47:193-198. https://doi.org/10.3901/JME.2011.19.193

3. Seibicke F, Müller H (2013) Good Things Need Some Time. Gear Solut 74-80

4. Antoniadis A (2012) Gear skiving - CAD simulation approach. CAD Comput Aided Des 44:611616. https://doi.org/10.1016/j.cad.2012.02.003

5. Kobialka C (2013) Contemporary gear pre-machining solutions. Gear Solut 43-49

6. Spath D, Hühsam A (2002) Skiving for high-performance machining of periodic structures. CIRP Ann - Manuf Technol 51:91-94. https://doi.org/10.1016/S0007-8506(07)61473-5

7. Guo Z, Mao SM, Li XE, Ren ZY (2016) Research on the theoretical tooth profile errors of gears machined by skiving. Mech Mach Theory 97:1-11. https://doi.org/10.1016/j.mechmachtheory.2015.11.001

8. Stadtfeld HJ (2014) Power skiving of cylindrical gears on different machine platforms. Gear Tech $52-62$

9. Sjoo S (2018) Tool and a cutting insert for power skiving 
10. Marx H, Vogel O (2012) Robust Method for Skiving and Corresponding Apparatus Comprising a Skiving Tool.pdf

11. Chen XC, Li J, Lou BC (2013) A study on the design of error-free spur slice cutter. Int J Adv Manuf Technol 68:727-738. https://doi.org/10.1007/s00170-013-4794-3

12. Chen XC, Li J, Zou Y, Wang P (2014) A study on the grinding of the major flank face of errorfree spur slice cutter. Int J Adv Manuf Technol 72:425-438. https://doi.org/10.1007/s00170-014-56269

13. Guo E, Hong R, Huang X, Fang C (2016) A novel power skiving method using the common shaper cutter. Int J Adv Manuf Technol 83:157-165. https://doi.org/10.1007/s00170-015-7559-3

14. Guo E, Hong R, Huang X, Fang C (2015) A correction method for power skiving of cylindrical gears lead modification. J Mech Sci Technol 29:4379-4386. https://doi.org/10.1007/s12206-0150936-X

15. Guo E, Hong R, Huang X, Fang C (2015) Research on the cutting mechanism of cylindrical gear power skiving. Int J Adv Manuf Technol 79:541-550. https://doi.org/10.1007/s00170-015-6816-9

16. Marx H, Vogel O (2015) Semi-completing skiving method and device having corresponding skiving tool for executing a semi-completing skiving method. 2:US009199323B2

17. Kreschel J, Kobialka C (2013) Method of generating gear teeth, and a gear-cutting machine that is operable according to said method. 1

18. Guo Z, Mao SM, Huyan L, Duan DS (2018) Research and improvement of the cutting performance of skiving tool. Mech Mach Theory 120:302-313. https://doi.org/10.1016/j.mechmachtheory.2017.08.004

19. Uriu K, Osafune T, Murakami T, et al (2017) Effects of shaft angle on cutting tool parameters in internal gear skiving. J Mech Sci Technol 31:5665-5673. https://doi.org/10.1007/s12206-017-1107-z 20. Tsai CY, Lin PD (2017) Gear manufacturing using power-skiving method on six-axis CNC turnmill machining center. Int J Adv Manuf Technol 1-15. https://doi.org/10.1007/s00170-017-1154-8

21. Guo Z, Mao SM, Du XF, Ren ZY (2017) Influences of tool setting errors on gear skiving accuracy. Int J Adv Manuf Technol 91:3135-3143. https://doi.org/10.1007/s00170-017-9988-7

22. Ren Z, Fang Z, Kobayashi G, et al (2020) Influence of tool eccentricity on surface roughness in gear skiving. Precis Eng 63:170-176. https://doi.org/10.1016/j.precisioneng.2020.02.007

23. Yang Y, Huang X, Yu C, et al (2019) Influence and correction for gear accuracy with geometric location errors in power skiving. Comput Integr Manuf Syst 25:1101-1111

24. Ding S, Huang X, Yu C, Liu X (2016) Identification of different geometric error models and definitions for the rotary axis of five-axis machine tools. Int. J. Mach. Tools Manuf. 100:1-6

25. Fang C, Gong J, Guo E, et al (2015) Analysis and Compensation for Gear Accuracy with Setting Error in Form Grinding. Adv Mech Eng 7:. https://doi.org/10.1155/2014/309148

26. (2013) ISO 1328:1: Cylindrical Gears - ISO System of Flank Tolerance Classification - Part 1: Definitions and Allowable Values of Deviations Relevant to Flanks of Gear Teeth. ISO, British Standards

27. Tang J, Nie J, Wang Z (2012) Reverse correction of spiral bevel gear HFT method. J Cent South Univ (Science Technol 43:2142-2149

28. Shih YP, Chen SD (2012) A flank correction methodology for a five-axis CNC gear profile grinding machine. Mech Mach 47:31-45. https://doi.org/10.1016/j.mechmachtheory.2011.08.009 